\title{
Nilpotent blocks of quasisimple groups for the prime two ${ }^{1}$
}

\author{
Jianbei An and Charles W. Eaton
}

\begin{abstract}
We examine nilpotency amongst blocks of positive defect of the quasisimple groups for the prime 2. We show that every nilpotent block of a quasisimple group has abelian defect groups, and prove a conjecture of Puig concerning the recognition of nilpotent blocks in the case of quasisimple groups. Explicit characterisations of nilpotent blocks are given for the classical, alternating and sporadic simple groups.
\end{abstract}

\section{Introduction}

Let $G$ be a finite group and $k$ an algebraically closed field of characteristic $p$. A block $B$ of $k G$ with defect group $D$ is said to be nilpotent if for each $Q \leq D$ and each block $b_{Q}$ of $C_{G}(Q)$ with Brauer correspondent $B$ we have that $N_{G}\left(Q, b_{Q}\right) / C_{G}(Q)$ is a $p$-group, where $N_{G}\left(Q, b_{Q}\right)$ is the stabilizer of $b_{Q}$ under conjugation in $N_{G}(Q)$. In the case of the principal block $B_{0}, D$ is a Sylow $p$-subgroup of $G$ and $N_{G}\left(Q, b_{Q}\right)=N_{G}(Q)$ for each $Q \leq D$, so that $B_{0}$ is nilpotent if and only if $G$ is $p$-nilpotent (i.e., $G$ has a normal p-complement).

Nilpotent blocks were in introduced in [10] and their structure fully determined in [26], where (amongst other things) it is shown that a nilpotent block is isomorphic to a full matrix algebra over the group algebra of a defect group. It follows that when $B$ is a nilpotent block, every block $b_{Q}$ of $C_{G}(Q)$ with $b_{Q}^{G}=B$, where $Q$ is a $p$-subgroup of $G$ has just one isomorphism class of simple modules.

Whilst the structure of nilpotent blocks is well-understood, there does not appear to be a great diversity of examples known either of nilpotent blocks themselves or of blocks which are 'almost' nilpotent. It is even the case that (to the authors' knowledge) there are no known examples of non-nilpotent blocks with just one simple module whose defect groups are involved in non-abelian simple groups. It is also interesting to find sufficient conditions for nilpotency of a block, as exemplified by the conjecture of Puig considered in this paper.

In this paper and in [6], which deals with the odd primes, we are concerned with two problems relating to nilpotent blocks of quasisimple groups. One is to determine the nilpotent blocks, the other is to establish means of determining when a block is nilpotent. We regard the second as the source of the main achievement in this paper, in that we verify a conjecture of Puig for quasisimple groups.

Note that blocks of defect zero are nilpotent, and the determination of the finite simple groups possessing a nilpotent block was completed in [17]. We therefore consider only the occurrence of nilpotent blocks of positive defect of the quasisimple groups. We also note that every block with defect groups of order two is nilpotent, so we are not concerned with this case either.

Explicit characterizations of nilpotent blocks are obtained for the classical groups, and these are used to prove:

\footnotetext{
${ }^{1}$ The first author is supported by the Marsden Fund (of New Zealand), via award number UOA 0721 and the second author is supported by a Royal Society University Research Fellowship
} 
Theorem 1.1 Let $G$ be a finite quasisimple group and let $B$ be a nilpotent 2-block of $G$. Then $B$ has abelian defect groups.

Results of this type already follow in special cases from, for example, [9], in which it is shown that the Frobenius category of a block of a symmetric, general linear or unitary group is equivalent to the Frobenius category of the principal block of a related group (and it is easy to determine when such a group is $p$-nilpotent).

In a recent paper of Malle and Navarro [24], it has been shown that if $B$ is a $p$ block of a quasisimple group $G$ which is not a faithful block of the double cover of an alternating group $A_{n}$ for $n \geq 14$, and is not a quasi-isolated block of an exceptional group of Lie type for $p$ a bad prime, and if every irreducible character of height zero has the same degree, then $B$ has abelian defect groups. In this case it follows that nilpotent blocks have abelian defect groups.

With regards to the recognition of nilpotent groups, we consider the following conjecture of Puig. For a finite dimensional $k$-algebra $A$, write $l(A)$ for the number of isomorphism classes of simple modules.

Conjecture 1.2 (Puig) Let $B$ be a block of a finite group $G$. Then $B$ is nilpotent if and only if $l\left(b_{Q}\right)=1$ for each p-subgroup $Q$ and each block $b_{Q}$ of $C_{G}(Q)$ with Brauer correspondent $B$.

The necessary condition for nilpotency is well-known. The converse is known for blocks with abelian defect groups (see [27]), and is also known to be a consequence of Alperin's weight conjecture (see [31]).

We prove:

Theorem 1.3 Let $G$ be a finite quasisimple group and let $B$ be a 2-block of $G$. Then $B$ is nilpotent if and only if $l\left(b_{Q}\right)=1$ for each 2-subgroup $Q$ and each block $b_{Q}$ of $C_{G}(Q)$ with $\left(b_{Q}\right)^{G}=B$.

Our strategy is to show that for the quasisimple groups of Lie type, every 2-block either has abelian defect groups (in which case the theorem holds by [27]) or $l\left(b_{Q}\right)>1$ for some block $b_{Q}$ with $b_{Q}^{G}=B$, where $Q$ is a $p$-subgroup of $G$. For the sporadic simple groups and the alternating groups (and their covers) each block $B$ is either nilpotent or $l(B)>1$.

The main part of the paper concerns the representation theory of finite groups of Lie type in non-defining characteristic, and makes use of an examination of subpairs of blocks of classical groups similar to that given in [14]. For these groups the failure of nilpotency of blocks with nonabelian defect groups is determined quite explicitly. The exceptional groups of Lie type are then treated by examination of the centralizer of an element of the centre of a defect group, and the results for the classical groups applied.

Before continuing we make a justification for the methods used in the paper. There are very elegant methods for the analysis of blocks of groups of Lie type involving the reduction to the theory of unipotent blocks (such as are used in [24]). These reductions respect nilpotency and so may be used to determine the nilpotent blocks of the finite groups of Lie type in most cases, and to show that nilpotent blocks of such groups must have abelian defect groups. We are informed that Kessar and Malle are near to 
completing the classification of quasi-isolated blocks, which together with the theory of unipotent blocks would allow an alternative proof that nilpotent blocks of quasisimple groups have abelian defect groups. However, to show that Puig's conjecture holds for blocks of quasisimple groups, it is necessary to use methods such as those of Fong and Srinivasan which allow a wider and more detailed analysis. In particular in order to check Puig's conjecture it is necessary to consider non-nilpotent blocks and to show the existence of a subpair with at least two simple modules, information which as far as we are aware is not yet known to be preserved under the reduction to unipotent and quasi-isolated blocks.

\section{Notation and general results}

Let $G$ be a finite group and $p$ a prime. Let $k$ be an algebraically closed field of characteristic $p$. Write $\operatorname{Blk}(G)$ for the set of blocks of $k G$ and denote by $B_{0}(G)$ the principal block of $G$.

Let $B$ be a $p$-block of a finite group $G$. A $B$-subgroup is a $\operatorname{subpair}\left(Q, b_{Q}\right)$, where $Q$ is a $p$-subgroup of $G$ and $b_{Q}$ is a block of $Q C_{G}(Q)$ with Brauer correspondent $\left(b_{Q}\right)^{G}=B$. The $B$-subgroups with $|Q|$ maximized are called the Sylow $B$-subgroups, and they are the $B$-subgroups for which $Q$ is a defect group for $B$. We will usually write $D(B)$ for a defect group of $B$ when one may be chosen freely.

A useful result, which follows from $[1,4.21]$, is the following:

Proposition 2.1 Let $B$ be a block of a finite group $G$. Suppose a defect group $D$ of $B$ is abelian. Then $B$ is nilpotent if and only if $N_{G}\left(D, b_{D}\right)=C_{G}(D)$, where $\left(D, b_{D}\right)$ is a Sylow B-subgroup.

We have the following lemma by [22, Proposition 6.5]:

Lemma 2.2 Let $N$ be a normal subgroup of a finite group $G$ such that $G / N$ is a pgroup. Suppose that $B$ is a block of $G$ and that $b \in \operatorname{Blk}(N)$ is covered by $B$. Then $B$ is nilpotent if and only if $b$ is nilpotent.

Recall that for $N \triangleleft G$, a block $B$ of $G$ is said to dominate the block $\bar{B}$ of $G / N$ if the inflation to $G$ of a simple $k \bar{G}$-module in $\bar{B}$ lies in $B$.

Let $B$ be a block of $G$ and $Z \leq O_{p}(Z(G))$. Let $\bar{B}$ be the unique block of $\bar{G}=G / Z$ dominated by $B$. If $\left(Q, b_{Q}\right)$ is a $B$-subgroup with $Z \leq Q$, then $C_{\bar{G}}(\bar{Q}) / \overline{C_{G}(Q)}$ is a $p$-group, and by $[31$, Lemma 1$]$ there is a unique $\bar{B}$-subgroup $\left(\bar{Q}, b_{\bar{Q}}\right)$ corresponding to $\left(Q, b_{Q}\right)$ (where $b_{\bar{Q}}$ is dominated by the unique block of the preimage in $G$ of $C_{\bar{G}}(\bar{Q})$ covering $b_{Q}$ ), and every $\bar{B}$-subgroup may be expressed in this way. It is clear that $l\left(b_{Q}\right)=l\left(b_{\bar{Q}}\right)$ in each case, so we have:

Lemma 2.3 Let $B$ be a block of $G$ and $Z \leq O_{p}(Z(G))$. Let $\bar{B}$ be the unique block of $\bar{G}=G / Z$ dominated by $B$. If $l\left(b_{Q}\right)=1$ for each $B$-subgroup $\left(Q, b_{Q}\right)$, then $l\left(b_{\bar{Q}}\right)=1$ for each $\bar{B}$-subgroup $\left(\bar{Q}, b_{\bar{Q}}\right)$. 
Lemma 2.4 Let $B$ be a 2-block of a finite group $K$, and let $(P, g) \leq(R, b)$ be $B$ subgroups with $P \leq Z(R)$ and let $Z \leq O_{2}\left(Z\left(C_{K}(P)\right)\right)$. Let $D(g)$ be a defect group of g. Suppose $y \in N_{C_{K}(P)}(R, b) \backslash C_{K}(R)$ for some $y$ with $|y|=3$. Suppose also that either

(i) $D(g) \cong \mathbb{Z}_{2^{c}} \backslash \mathbb{Z}_{2}$ for some integer $c \geq 2$ and $R \cong Z(D(g)) Q_{8}$, or

(ii) $D(g) / Z \leq D_{2^{c+1}}$ and $\bar{R}:=(R Z) / Z \cong \mathbb{Z}_{2} \times \mathbb{Z}_{2}$.

Then $\ell(g) \geq 2$.

Proof: Note that since $R C_{K}(R) \leq C_{K}(P)$, we may without loss of generality assume $R \leq D(g)$.

Suppose that $D(g) \cong \mathbb{Z}_{2^{c}}\left\langle\mathbb{Z}_{2}\right.$, where $c \geq 2$, and $R \cong Z(D(g)) Q_{8}$. We apply [21] to $g$. In [21] a set of subgroups $\mathfrak{Q}$ of $D(g)$ is defined and a system of conjugacy class representatives given. By $[21, \operatorname{Lemma}(2 . \mathrm{B})] R \in \mathfrak{Q}$. We have $\left[N_{K}(R, b): C_{K}(R) R\right] \geq 3$. Hence $R$ is one of the groups identified in part (ii) of [21, Lemma (2.B)], and so case (bB) of [21] cannot occur (see [21, p.533]), and by [21, Propositions (7.G), (14.E), (14.G)], $\ell(g) \geq 2$.

Suppose $D(g) / Z \leq D_{2^{c+1}}$ and $(R Z) / Z \cong \mathbb{Z}_{2} \times \mathbb{Z}_{2}$, so that $D(g) / Z$ is $\bar{R}$ or is dihedral, as $\bar{R} \leq D(g) / Z$. Let $\bar{g}$ and $\bar{b}$ be the unique blocks of $C_{G}(P) / Z$ and $C_{G}(R) / Z$ such that $\bar{g}$ and $\bar{b}$ are dominated by $g$ and $b$, respectively, so that $D(\bar{g})=D(g) / Z$ and $\ell(g)=\ell(\bar{g})$. By [30], $\ell(\bar{g})$ is the number of 2-weights of $\bar{g}$. If $D(\bar{g})=\bar{R}$, then $\bar{y} \in N_{K / Z}(D(\bar{g}), \bar{b})$ with $\bar{y}=y Z$ and $\bar{g}$ has three weights $\left(D(\bar{g}), \psi_{i}\right)$, where each $\psi_{i}$ is an extension of the canonical character $\bar{\theta}$ of $\bar{b}$ to $N_{K / Z}(D(\bar{g}), \bar{b})$. If $D(\bar{g})$ is dihedral, then $N_{K / Z}(\bar{R}, \bar{b}) / C_{K / Z}(\bar{R})$ is isomorphic to the symmetric group $S_{3}$ and $\bar{g}$ has at least two weights $(\bar{R}, \psi),(D(\bar{g}), \bar{\psi})$, where $\psi$ is the extension of $\bar{\theta}$ to $N_{K / Z}(\bar{R}, \bar{b})$ with $\psi(1)=2 \bar{\theta}(1)$, and $\bar{\psi}$ is the canonical character of the root block $b_{D(\bar{g})}$ of $\bar{g}$. Thus $\ell(g)=\ell(\bar{g}) \geq 2$.

The next lemma follows from [31, Lemma 2].

Lemma 2.5 Let $Z$ be a central p-subgroup of a finite group $G, B \in \operatorname{Blk}(G)$ and $\bar{B}$ the block of $\bar{G}:=G / Z$ dominated by $B$. Then $B$ is nilpotent if and only if $\bar{B}$ is nilpotent.

Let $Z$ be a central $p^{\prime}$-subgroup of a finite group $G$, and write $\bar{H}=H Z / Z$, where $H \leq G$. Let $\bar{B} \in \operatorname{Blk}(\bar{G})$. There is a unique block $B \in \operatorname{Blk}(G)$ dominating $\bar{B}$. By [25, Theorem 5.8.8], $\operatorname{Irr}(B)=\operatorname{Irr}(\bar{B})$ and if $D$ is a defect group of $B$, then $D Z / Z \cong D$ is a defect group of $\bar{B}$.

If $Q$ is a $p$-subgroup of $G$, then $C_{\bar{G}}(\bar{Q})=C_{G}(Q) / Z$ (since $Z$ is a central $p^{\prime}$-subgroup). Let $\left(\bar{Q}, b_{\bar{Q}}\right)$ be a $\bar{B}$-subgroup. Then $\bar{Q}=Q Z / Z$ for a unique $p$-subgroup $Q$ of $G$. Since $C_{\bar{G}}(\bar{Q})=C_{G}(Q) / Z$, we may consider the unique subpair $\left(Q, b_{Q}\right)$ with $b_{Q}$ dominating $b_{\bar{Q}}$, which we call the Brauer pair dominating $\left(\bar{Q}, b_{\bar{Q}}\right)$.

The next lemma, proved in [6], says that $\left(Q, b_{Q}\right)$ must be a $B$-subgroup, and that dominance of subpairs respects the usual partial order on $B$-subgroups:

Lemma 2.6 Let $Z$ be a central $p^{\prime}$-subgroup of a finite group $G$, and let $\left(\bar{Q}, b_{\bar{Q}}\right)$ and $\left(\bar{P}, b_{\bar{P}}\right)$ be $\bar{B}$-subgroups, where $\bar{B}$ is the block of $\bar{G}$ dominated by $B$. Suppose $\left(Q, b_{Q}\right)$ and $\left(P, b_{P}\right)$ are subpairs of $G$ dominating $\left(\bar{Q}, b_{\bar{Q}}\right)$ and $\left(\bar{P}, b_{\bar{P}}\right)$, respectively. Then $\left(\bar{Q}, b_{\bar{Q}}\right) \leq$ $\left(\bar{P}, b_{\bar{P}}\right)$ if and only if $\left(Q, b_{Q}\right) \leq\left(P, b_{P}\right)$. In particular, $\left(Q, b_{Q}\right)$ is a B-subgroup.

As a consequence (see $[6])$ : 
Proposition 2.7 Let $G$ be a finite group, $Z \leq Z(G)$ and $\bar{G}=G / Z$. Suppose $\bar{B} \in$ $\operatorname{Blk}(\bar{G})$ and $B \in \operatorname{Blk}(G)$ dominating $\bar{B}$. Then $\bar{B}$ is nilpotent if and only if $B$ is nilpotent.

\section{The symmetric and alternating groups}

Write $\hat{S}_{n}$ for the double cover of the symmetric group $S_{n}$. Then the 2-blocks of $\hat{S}_{n}$ and of $S_{n}$ are in one-to-one correspondence under the natural epimorphism, and the block corresponding to a nilpotent block is nilpotent (by [31, Lemma 2]). Hence it suffices to consider $S_{n}$ (except for the exceptional cases $n=6,7$, which we treat separately).

Proposition 3.1 Let $G=S_{n}$ with $n \geq 2$. If $D$ is a non-trivial defect group of a nilpotent 2-block, then $D$ is generated by a transposition and $n=2+m(m+1) / 2$ for some positive integer $m$. Conversely, if $n=2+m(m+1) / 2$ for some positive integer $m$, then $S_{n}$ possesses precisely one nilpotent 2-block.

Note that this result follows from [9, 2.B.4], which shows that the Frobenius category of a block of a symmetric group is equivalent to the Frobenius category of the principal block of a (possibly smaller) symmetric group. We give our own proof here.

Proof: Let $B$ be a nilpotent block with non-trivial defect group $D$. Let $y \in D$ be an involution. Suppose that $y$ is a product of $t>1$ disjoint transpositions and fixes every other point. Then $C_{S_{n}}(y) \cong\left(\mathbb{Z}_{2} 2 S_{t}\right) \times S_{n-2 t}$, and in particular $C_{S_{n}}(y)$ contains a normal elementary abelian 2 -group $R$ generated by $t$ disjoint transpositions. Then $R \leq O_{2}\left(C_{S_{n}}(y)\right)$, and so is contained in every defect group of $C_{S_{n}}(y)$. Hence by a well-known property of the Brauer correspondence $R$ is contained in a conjugate of $D$. So $D$ contains an elementary subgroup $Q$ of order 4 such that $C_{S_{n}}(Q) \cong Q \times S_{n-4}$ and $N_{S_{n}}(Q) \cong S_{4} \times S_{n-4}$. Now every 2-block of $C_{S_{n}}(Q)$ is $N_{S_{n}}(Q)$-stable. Since $\left[N_{S_{n}}(Q): C_{S_{n}}(Q)\right]=6$, it follows that $B$ cannot be nilpotent. Hence $D$ is generated by a transposition.

Note that every block with defect groups of order two is nilpotent. The blocks of $S_{n}$ with defect group $D$ generated by a transposition are in one-to-one correspondence with the blocks of $N_{S_{n}}(D) \cong D \times S_{n-2}$ with defect group $D$, and hence in one-to-one correspondence with the blocks of defect zero of $S_{n-2}$. Since a Young diagram for $S_{n-2}$ is a 2 -core precisely when $n-2=m(m+1) / 2$ for some positive integer $m$, and in this case there is precisely one such diagram, the result follows by Nakayama's Conjecture.

Corollary 3.2 Let $G=A_{n}$ with $n \geq 3$. Then $G$ possesses no nilpotent 2-block of positive defect.

Proof: This follows immediately from Proposition 3.1 and Lemma 2.2, since the defect groups of nilpotent blocks of positive defect of $S_{n}$ do not lie in $A_{n}$.

Corollary 3.3 Let $G$ be a quasisimple group with $G / Z(G) \cong A_{n}$ for some $n$. Then $G$ has no nilpotent 2-block with non-central defect groups. 
Proof: The exceptional covers $6 . A_{6}$ and $6 . A_{7}$ follow by observing that every block with non-central defect groups has at least two irreducible Brauer characters (see [15]). The result then follows from Corollary 3.2 and Lemma 2.5.

Proposition 3.4 Let $B$ be a 2-block of a quasisimple group $G$ such that $G / Z(G) \cong A_{n}$ for some $n$. Then $B$ is nilpotent if and only if $l\left(b_{Q}\right)=1$ for each $B$-subgroup $\left(Q, b_{Q}\right)$.

Proof: It suffices to show the sufficient condition for nilpotency. Let $D$ be a defect group for $B$. The exceptional covers $6 . A_{6}$ and $6 . A_{7}$ follow by observing that every block with non-central defect groups has at least two irreducible Brauer characters (see [15]). Hence we may suppose that $G$ is simple or a double cover of some $A_{n}$. By Lemmas 2.3 and 2.5 it suffices to consider the case $G \cong A_{n}$. Let $G \leq E \cong S_{n}$, and let $C$ be the unique 2-block of $E$ covering $B$.

By, for example, [18, 6.2.2], $l(C)=p(w)$, where $w$ is the weight of $C$ and $p(w)$ is the number of partitions of $w$. If $|D|=2$, then $B$ is nilpotent. Suppose that $|D| \geq 4$, so $C$ has defect at least two. Then $w \geq 2$, and $l(C)=p(w) \geq 2$. Hence we must have $l(B)>1$, and the result follows.

\section{Sporadic groups and their covers}

In this section we determine the nilpotent blocks with non-central defect groups of quasisimple groups $G$ where $G / Z(G)$ is one of the 26 sporadic simple groups. Note that due to Lemma 2.5 it suffices to consider the case $Z(G)$ has odd order.

We show that each 2-block of such a group (where $|Z(G)|$ is odd) with defect greater than one has at least two irreducible Brauer characters. In most cases this may be seen using the library in [15]. As a consequence every nilpotent block of any quasisimple group $G$ with $G / Z(G)$ sporadic has abelian defect groups.

Proposition 4.1 Let $G$ be a quasisimple group such that $G / Z(G)$ is a sporadic simple group. Let $B$ be a 2-block of $G$ and let $D$ be a defect group of $B$. If $|D| \geq 4$, then $l(B)>1$.

Proof: It suffices to consider the case $|Z(G)|$ odd. If $D$ is cyclic, then the result follows from the theory of blocks with cyclic defect groups. In the following table we list the numbers of irreducible Brauer characters in 2-blocks with non-cyclic defect groups, along with a reference. A ${ }^{*}{ }^{\prime}$, will be used to denote a faithful block in a group with non-trivial centre. The result then follows from examination of the table.

Note that since $|Z(G)|$ is odd, if $Q$ is a 2-subgroup of $G$, then

$$
\left.C_{G / Z(G)}(Q Z(G)) / Z(G)\right) \cong C_{G}(Q) / Z(G) .
$$

We treat each of the cases for which the number of simple $k G$-modules in each block is not currently given either in [15] or other references of which we are aware.

Suppose $G \cong L y$. Then $G$ as two blocks of positive defect by [28], the principal block $B_{0}$ and a block $B_{1}$ of defect seven, and $k\left(B_{0}\right):=\left|\operatorname{Irr}\left(B_{0}\right)\right|=25$. But $G$ has one 
conjugate class of involutions and one of elements of order 4 , and two of order 8 , so $k\left(B_{0}\right)=l\left(B_{0}\right)+l\left(b_{1}\right)+l\left(b_{2}\right)+l\left(b_{3}\right)+l\left(b_{3}^{\prime}\right)$, where $b_{i}=B_{0}\left(C_{G}\left(z_{i}\right)\right)$ with $\left|z_{i}\right|=2^{i}$ and $b_{3}^{\prime}=B_{0}\left(C_{G}\left(z_{3}^{\prime}\right)\right)$ such that $z_{3}^{\prime}$ is another element of order 8 which is non-conjugate to $z_{3}$. Now $C_{G}\left(z_{1}\right)=\hat{A}_{11}, C_{G}\left(z_{2}\right)=\left(4 \circ \hat{A}_{7}\right) .2, C_{G}\left(z_{3}\right)=8 \circ \hat{A}_{5}$ and $C_{G}\left(z_{3}^{\prime}\right)=8 . A_{4}$, so

$$
l\left(b_{1}\right)=l\left(B_{0}\left(A_{11}\right)\right), \quad l\left(b_{2}\right)=l\left(B_{0}\left(S_{7}\right)\right), \quad l\left(b_{3}\right)=l\left(B_{0}\left(A_{5}\right)\right) \quad \text { and } \quad l\left(b_{3}^{\prime}\right)=3 .
$$

The Brauer characters of $A_{11}$ and $A_{5}$ are given by [19], and a calculation shows that $l\left(B_{0}\left(A_{11}\right)\right)=7$ and $l\left(B_{0}\left(A_{5}\right)\right)=3$. Since $l\left(B_{0}\left(S_{7}\right)\right)$ is the number of partitions of the weight 3 , it follows that $l\left(b_{2}\right)=3$, and $l\left(B_{0}\right)=25-7-3-3-3=9$. Since $G$ has 10 blocks of defect 0 and 27 regular classes, it follows that $l\left(B_{1}\right)=27-9-10=8$.

\begin{tabular}{|c|c|c|c|}
\hline$G / Z(G)$ & $|D|$ & $\ell(B)$ & reference \\
\hline$M_{11}$ & $2^{4}$ & 3 & {$[15]$} \\
\hline$M_{12}$ & $2^{6} / 2^{2}$ & $3 / 3$ & {$[15]$} \\
\hline$M_{22}$ & $2^{7} / 2^{7} / 2^{7}$ & $7 / 5^{*} / 5^{*}$ & {$[15]$} \\
\hline$M_{23}$ & $2^{7}$ & 9 & {$[15]$} \\
\hline$M_{24}$ & $2^{10}$ & 13 & {$[15]$} \\
\hline$J_{1}$ & $2^{3}$ & 5 & {$[15]$} \\
\hline$J_{2}$ & $2^{7} / 2^{2}$ & $7 / 3$ & {$[15]$} \\
\hline$J_{3}$ & $2^{7} / 2^{7} / 2^{7}$ & $10 / 10^{*} / 10^{*}$ & {$[15]$} \\
\hline$J_{4}$ & $2^{21}$ & 22 & {$[8]$} \\
\hline$H S$ & $2^{9} / 2^{2}$ & $6 / 3$ & {$[15]$} \\
\hline$M c L$ & $2^{7} / 2^{7} / 2^{7}$ & $8 / 8^{*} / 8^{*}$ & {$[15]$} \\
\hline Suz & $2^{13} / 2^{3} / 2^{13} / 2^{13}$ & $14 / 3 / 14^{*} / 14^{*}$ & {$[15]$} \\
\hline$L y$ & $2^{8} / 2^{7}$ & $9 / 8$ & {$[28]$} \\
\hline $\mathrm{He}$ & $2^{10} / 2^{3}$ & $11 / 3$ & {$[15]$} \\
\hline$R u$ & $2^{14} / 2^{2}$ & $6 / 3$ & {$[15]$} \\
\hline$O^{\prime} N$ & $2^{9} / 2^{3} / 2^{9} / 2^{9}$ & $5 / 3 / 5^{*} / 5^{*}$ & {$[15]$} \\
\hline $\mathrm{Co}_{3}$ & $2^{10} / 2^{3}$ & $10 / 5$ & {$[15]$} \\
\hline $\mathrm{CO}_{2}$ & $2^{18}$ & 12 & {$[15]$} \\
\hline $\mathrm{Co}_{1}$ & $2^{21} / 2^{3}$ & $26 / 2$ & {$[7]$} \\
\hline$F i_{22}$ & $2^{17} / 2^{17} / 2^{17}$ & $14 / 11^{*} / 11^{*}$ & {$[15]$} \\
\hline$F i_{23}$ & $2^{18} / 2^{3}$ & $20 / 2$ & {$[15]$} \\
\hline$F i_{24}^{\prime}$ & $2^{21} / 2^{2} / 2^{3} / 2^{21} / 2^{21} / 2^{3} / 2^{3}$ & $33 / 3 / 3 / 22^{*} / 22^{*} / 3^{*} / 3^{*}$ & {$[5]$} \\
\hline$T h$ & $2^{15}$ & 18 & {$[29]$} \\
\hline$H N$ & $2^{14} / 2^{4}$ & $17 / 3$ & {$[15]$} \\
\hline$F_{2}=B$ & $2^{41} / 2^{3}$ & $25 / 2$ & \\
\hline$F_{1}=M$ & $2^{46} / 2^{4}$ & $52 / 3$ & \\
\hline
\end{tabular}

Table 1: Numbers of irreducible Brauer characters in 2-blocks with non-cyclic, noncentral defect groups of covering groups of sporadic simple groups 
Suppose $G \cong T h$. Then $G$ has three 2-blocks of defect zero and the principal block (see [29]). By [12] $l(G)=21$, so $l\left(B_{0}(G)\right)=18$.

Suppose $G \cong 3 . F i_{24}^{\prime}$. By [5] there is a unique block of maximal defect and a unique block with $D_{8}$ defect groups covering each block of $Z(G)$. Further, there is a non-faithful block with Klein-four defect groups. The non-faithful blocks have 33 or 3 simple $k G$-modules. Now (see [23]), if $D \cong D_{8}$ is a defect group, then $(D Z(G) / Z(G)) C_{G / Z(G)}(D Z(G) / Z(G)) \cong D_{8} \times S p_{6}(2)$, so $D C_{G}(D) \cong Z(G) \times D \times S p_{6}(2)$ and the blocks with defect group $D$ have the same number of simple $k G$-modules, i.e., three. By consideration of character degrees in [12], $G$ as no faithful blocks of defect one. Further, there are 25 simple $k G$-modules covering each faithful simple $k Z(G)$ module. So the faithful blocks of maximal defect each have 22 simple modules.

Suppose $G \cong F_{2}$, the baby monster. It is clear from [12] that $G$ has no 2-blocks of defect zero. By [23] $G$ has two blocks of positive defect, the principal block $B_{0}(G)$ and another $B_{1}$ with defect group $D_{8}$, where $l\left(B_{1}\right)=2$. Since $l(G)=27$, we have $l\left(B_{0}(G)\right)=25$.

Suppose $G \cong M$, the monster group. By [23] $G$ has two blocks of positive defect: the principal block $B_{0}(G)$ and one $B_{1}$ with semi-dihedral defect groups of order 16 . From [12] we see that $G$ has three 2-blocks of defect zero. By [20] we have $l\left(B_{1}\right)=3$. Since $l(G)=58$, it follows that $l\left(B_{0}(G)\right)=52$.

Corollary 4.2 Let $G$ be a quasisimple group such that $G / Z(G)$ is a sporadic simple group. Then $G$ has no nilpotent block with defect group $D$ such that $D / O_{p}(Z(G))$ has of order greater than two. In particular, every nilpotent block of $G$ has abelian defect groups.

Corollary 4.3 Let $G$ be a quasisimple group such that $G / Z(G)$ is a sporadic simple group. Then $B$ is nilpotent if and only if $l\left(b_{Q}\right)=1$ for each $B$-subgroup $\left(Q, b_{Q}\right)$.

\section{$5 \quad$ Notation for classical groups}

Let $V$ be a linear, unitary, non-degenerate orthogonal or symplectic space over the field $\mathbb{F}_{q}$, where $q=r^{a}$ for some odd prime $r$. We will follow the notation of [4], [9], [13] and [14].

If $V$ is orthogonal, then there is a choice of equivalence classes of quadratic forms. Write $\eta(V)$ for the type of $V$ as defined in [14], so $\eta(V)=\eta=+$ or - . Let $\eta(V)=+$ if $V$ is linear and $\eta(V)=-$ if $V$ is unitary. If $V$ is non-degenerate orthogonal or symplectic, then denote by $I(V)$ the group of isometries on $V$ and let $I_{0}(V)=I(V) \cap$ $\mathrm{SL}(V)$.

If $V$ is symplectic, then $I(V)=I_{0}(V)=\operatorname{Sp}_{2 n}(q)$.

If $V$ is a $(2 n+1)$-dimensional orthogonal space, then $I(V)=\left\langle-1_{V}\right\rangle \times I_{0}(V)$ with $I_{0}(V)=\mathrm{SO}_{2 n+1}(q)$.

If $V$ is a $2 n$-dimensional orthogonal space, then $I(V)=O^{\eta}(V)=O_{2 n}^{\eta}(q)$ and $I_{0}(V)=\mathrm{SO}_{2 n}^{\eta}(q)$.

If $V$ is a $2 n$-dimensional non-degenerate orthogonal or symplectic space, then denote by $J_{0}(V)$ the conformal isometries of $V$ with square determinant. If $V$ is orthogonal 
of dimensional at least two, then write $D_{0}(V)$ for the special Clifford group of $V$ (cf. [14]).

Denote by $\mathrm{GL}^{+}(V)$ the general linear group $\mathrm{GL}(V)$ and $\mathrm{GL}^{-}(V)$ the unitary group $U(V)$.

Let $G=\mathrm{GL}^{\eta}(V)$ or $I(V)$. Write $\mathcal{F}_{q}=\mathcal{F}_{q}(G)$ for the set of (monic) polynomials serving as elementary divisors for semisimple elements of $G$ (cf. [4, p.6]). If $G=$ $\mathrm{GL}^{-}(V)$, then partition $\mathcal{F}_{q}$ as in [13] by writing:

$$
\begin{aligned}
& \mathcal{F}_{1}=\left\{\Delta \in \mathcal{F}_{q}: \Delta \text { irreducible, } \Delta \neq X, \Delta=\tilde{\Delta}\right\}, \\
& \mathcal{F}_{2}=\left\{\Delta \tilde{\Delta} \in \mathcal{F}_{q}: \Delta \text { irreducible, } \Delta \neq X, \Delta \neq \tilde{\Delta}\right\}
\end{aligned}
$$

where $\tilde{\Delta}$ is the monic irreducible polynomial whose roots are the $q$-th power of those of $\Delta$. If $G=I(V)$, then partition $\mathcal{F}_{q}$ as in [14] by writing:

$$
\begin{aligned}
& \mathcal{F}_{0}=\{X \pm 1\}, \\
& \mathcal{F}_{1}=\left\{\Delta \in \mathcal{F}_{q}-\mathcal{F}_{0}: \Delta \text { irreducible, } \Gamma \neq X, \Delta=\Delta^{*}\right\}, \\
& \mathcal{F}_{2}=\left\{\Delta \Delta^{*} \in \mathcal{F}_{q}-\mathcal{F}_{0}: \Delta \text { irreducible, } \Delta \neq X, \Delta \neq \Delta^{*}\right\},
\end{aligned}
$$

where $\Delta^{*}$ is the monic irreducible polynomial whose roots are the inverses of those of $\Delta$. Let $d_{\Gamma}$ be the degree of $\Gamma \in \mathcal{F}_{q}$, and define the reduced degree $\delta_{\Gamma}$ as in [4] and [14], so that $\delta_{\Gamma}=d_{\Gamma}$ if $G=\mathrm{GL}^{\eta}(V)$ or $\Gamma \in \mathcal{F}_{0}$, and $\delta_{\Gamma}=\frac{1}{2} d_{\Gamma}$ if $G=I(V)$ and $\Gamma \in \mathcal{F}_{1} \cup \mathcal{F}_{2}$.

For integers $c, m$, we write $2^{c} \| m$ when $2^{c} \mid m$ and $2^{c+1} \nmid m$, and we let $a \geq 2$ be the integer such that $2^{a+1} \|\left(q^{2}-1\right)$. Let $\epsilon= \pm 1$, chosen so that $2^{a} \mid(q-\epsilon)$. For each $\Gamma \in \mathcal{F}_{q}$, define $\epsilon_{\Gamma}=1$ when $G=\mathrm{GL}(V)$, otherwise define

$$
\epsilon_{\Gamma}=\left\{\begin{array}{ccc}
\epsilon & \text { if } & \Gamma \in \mathcal{F}_{0}, \\
-1 & \text { if } & \Gamma \in \mathcal{F}_{1}, \\
1 & \text { if } & \Gamma \in \mathcal{F}_{2}
\end{array}\right.
$$

Let $e_{\Gamma}$ be the multiplicative order of $\epsilon_{\Gamma} q^{\delta_{\Gamma}}$ modulo 4 . Thus we may write $e_{\Gamma} \delta_{\Gamma}=$ $e 2^{\alpha_{\Gamma}} \delta_{\Gamma}^{\prime}$ for some $\alpha_{\Gamma}$ and $\delta_{\Gamma}^{\prime}$ with odd $\delta_{\Gamma}^{\prime}$, where $e=e_{X-1}$.

Given a semisimple element $s \in G$, there is a unique orthogonal decomposition $V=\sum_{\Gamma \in \mathcal{F}_{q}} V_{\Gamma}(s)$, with $s=\prod_{\Gamma \in \mathcal{F}_{q}} s(\Gamma)$, where the $V_{\Gamma}(s)$ are nondegenerate subspaces of $V$ and $s(\Gamma) \in U\left(V_{\Gamma}(s)\right)$, $\mathrm{GL}\left(V_{\Gamma}(s)\right)$ or $I\left(V_{\Gamma}(s)\right)$ (depending on $G$ ) has minimal polynomial $\Gamma$. This is called the primary decomposition of $s$. Write $m_{\Gamma}(s)$ for the multiplicity of $\Gamma$ in $s(\Gamma)$. We have $C_{G}(s)=\prod_{\Gamma \in \mathcal{F}_{q}} C_{\Gamma}(s)$, where $C_{\Gamma}(s)=I\left(V_{\Gamma}(s)\right)$ or $\mathrm{GL}^{\epsilon_{\Gamma}}\left(m_{\Gamma}(s), q^{\delta_{\Gamma}}\right)$ as appropriate.

We will also be using the Deligne-Lusztig theory of characters of finite reductive groups, so we briefly review the relevant notation. An excellent source for this material is [11]. Let $\mathbf{G}$ be a connected reductive group over $\overline{\mathbb{F}}_{q}$ and let $F: \mathbf{G} \rightarrow \mathbf{G}$ be the Frobenius automorphism associated to the $\mathbb{F}_{q}$-structure of $\mathbf{G}$. Let $\left(\mathbf{G}^{*}, F^{*}\right)$ be dual to $(\mathbf{G}, F)$ with respect to a dual pair of $F$-stable (resp. $F^{*}$-stable) maximal tori $\left(\mathbf{T}, \mathbf{T}^{*}\right)$. We consider the finite group $G=\mathbf{G}^{F}$. The irreducible characters $\operatorname{Irr}(G)$ may be partitioned into rational series $\mathcal{E}(G,(t))$ indexed by $\left(\mathbf{G}^{*}\right)^{F^{*}}$-conjugacy classes $(t)$ of semisimple elements of $\left(\mathbf{G}^{*}\right)^{F^{*}}$. For $s$ a semisimple $p^{\prime}$-element of $\left(\mathbf{G}^{*}\right)^{F^{*}}$, write $\mathcal{E}_{p}(G,(s))$ for the union of the $\mathcal{E}(G,(s u))$ where $u$ is a $p$-element centralizing $s$. 


\section{$6 \quad$ Blocks of linear and unitary groups}

In Section 7 we will state a property which we will show holds for every 2-block of a classical group. Before doing this we gather some information about nilpotency of blocks of general linear and unitary groups which will be used repeatedly.

Suppose $G=\mathrm{GL}_{n}^{\eta}(q)=\mathrm{GL}^{\eta}(V)$ and $r$ is an odd prime, and let $B$ be a 2-block of $G$. Then $B=\mathcal{E}_{2}(G,(s))$ for some semisimple $2^{\prime}$-element $s \in G$. For convenience we denote $\mathrm{GL}^{\eta}(V)$ by $G(V)$ and $\mathrm{SL}^{\eta}(V)$ by $S(V)$.

We note that [9] already provides the means of showing that every nilpotent block of $\mathrm{GL}^{\eta}(V)$ has abelian defect groups. In particular by $[9,3.8]$ the Frobenius category of a block of $\mathrm{GL}^{\eta}(V)$ is equivalent to the Frobenius category of the principal block of a direct product of unitary and general linear groups, but every 2-nilpotent unitary or general linear group has abelian Sylow 2-subgroups. However, for later results we need to prove something stronger:

Theorem 6.1 Let $G=\mathrm{GL}^{\eta}(V)=\mathrm{GL}^{\eta}(n, q)$. Then the following are equivalent.

(a) $B$ is a nilpotent block of $G$.

(b) $m_{\Gamma}(s) \leq 1$ for all $\Gamma \in \mathcal{F}_{q}$.

(c) Let $\left(D, b_{D}\right)$ be a Sylow B-subgroup and $\theta$ the canonical character of $b_{D}$. Write $C=C_{G}(D)$. Then $\theta= \pm R_{T}^{C}(s)$ with $T=C_{C}(s)$ a torus of both $G$ and $C$, and $D=O_{2}(T)$. Here $R_{T}^{C}(s)$ is the Deligne-Lusztig generalized character.

In particular, if $B$ is nilpotent, then $D$ is abelian.

Proof: In this proof, to avoid confusion with notation we write $\mathbf{S}(n)$ for the symmetric group on $n$ letters.

Let $s=\prod_{\Gamma} s(\Gamma)$ be a primary decomposition, so that $V=\bigoplus_{\Gamma} V_{\Gamma}$ with $V_{\Gamma}=V_{\Gamma}(s)$ the underlying space of $s(\Gamma)$. Thus

$$
C_{G}(s)=\prod_{\Gamma} C_{\Gamma}, \quad C_{\Gamma}=\mathrm{GL}^{\epsilon_{\Gamma}}\left(m_{\Gamma}, q^{\delta_{\Gamma}}\right)
$$

with $m_{\Gamma}=m_{\Gamma}(s)$ and $C_{\Gamma}=C_{\Gamma}(s)$. We may suppose $D \in \operatorname{Syl}_{2}\left(C_{G}(s)\right)$, so that

$$
D=\prod_{\Gamma} D_{\Gamma}, \quad D_{\Gamma} \in \operatorname{Syl}_{2}\left(C_{\Gamma}\right)
$$

So $D$ is a direct product of iterated wreath product 2-groups. Write

$$
m_{\Gamma}=n_{\Gamma}+2^{c_{1}+1}+\cdots+2^{c_{t}+1}
$$

where $0 \leq c_{1}<\cdots<c_{t}$ and $n_{\Gamma}=0$ or 1 according as $m_{\Gamma}$ is even or odd.

Let $U_{\Gamma}$ be the underlying space of $\operatorname{GL}^{\eta}\left(d_{\Gamma}, q\right)$ and $W_{\Gamma}=U_{\Gamma} \perp U_{\Gamma}$ the underlying space of $\mathrm{GL}^{\eta}\left(2 d_{\Gamma}, q\right)$. Let

$$
W_{\Gamma}(i)=W_{\Gamma} \perp \cdots \perp W_{\Gamma} \quad\left(2^{c_{i}} \text { terms }\right)
$$


for $i>0$, and $W_{\Gamma}(0)=U_{\Gamma}$ or $W_{\Gamma}(0)=0$ according as $n_{\Gamma}=1$ or 0 .

Let $Y_{\Gamma}=\mathrm{GL}^{\epsilon_{\Gamma}}\left(2, q^{\delta_{\Gamma}}\right)$ be a regular subgroup of $G\left(W_{\Gamma}\right)=\mathrm{GL}^{\eta}\left(2 d_{\Gamma}, q\right)$ and $S_{\Gamma} \in$ $\operatorname{Syl}_{2}\left(Y_{\Gamma}\right)$. Then

$$
V_{\Gamma}=W_{\Gamma}(0) \perp W_{\Gamma}(1) \perp \cdots \perp W_{\Gamma}(t), \quad D_{\Gamma}=S_{0} \prod_{i \geq 1}\left(S_{\Gamma}\left\langle X_{2^{c_{i}}}\right)\right.
$$

where $S_{\Gamma} 2 X_{2^{c_{i}}} \leq\left(Y_{\Gamma}\right)^{2^{c_{i}}} \leq G\left(W_{\Gamma}(i)\right)$ for $i>1$, and $S_{0}=O_{2}\left(\mathrm{GL}^{\epsilon_{\Gamma}}\left(1, q^{\delta_{\Gamma}}\right)\right)$ or 1 according as $n_{\Gamma}=1$ or 0 , and $X_{2^{c_{i}}} \in \operatorname{Syl}_{2}\left(\mathbf{S}\left(2^{c_{i}}\right)\right)$.

For $i \geq 1$, set $E\left(S_{\Gamma} \succ X_{2^{c_{i}}}\right)=\left(S_{\Gamma}\right)^{2^{c_{i}}}$ to be the base subgroup of $S_{\Gamma} \succ X_{2^{c_{i}}}$ and let

$$
E(D):=\prod_{\Gamma} E\left(D_{\Gamma}\right), \quad E\left(D_{\Gamma}\right):=S_{0} \prod_{i \geq 1} E\left(S_{\Gamma} \prec X_{2^{c_{i}}}\right)=S_{0} \times\left(S_{\Gamma}\right)^{\left(m_{\Gamma}-n_{\Gamma}\right) / 2},
$$

so that $E(D) \triangleleft D$ and each component is either cyclic or equal to $S_{\Gamma}$ for some $\Gamma$. Thus $E(D)$ is abelian if and only if $D$ is abelian if and only if each $m_{\Gamma} \leq 1$ if and only if $C_{G}(s)$ is a maximal torus of $G$.

Let $H_{\Gamma} \triangleleft Y_{\Gamma}$ such that $H_{\Gamma} \cong \mathrm{SL}\left(2, q^{\delta_{\Gamma}}\right)$, so that $S_{\Gamma} \cap H_{\Gamma} \in \operatorname{Syl}_{2}\left(H_{\Gamma}\right)$ and $S_{\Gamma} \cap H_{\Gamma}$ is generalized quaternion. By $[3,(1 \mathrm{H})(\mathrm{a})], S_{\Gamma} \cap H_{\Gamma}$ contains a quaternion subgroup $Q_{\Gamma} \cong Q_{8}$ such that

$$
N_{H_{\Gamma}}\left(Q_{\Gamma}\right) / Q_{\Gamma}=\mathbb{Z}_{3} \quad \text { or } \quad \mathbf{S}(3)
$$

according as $q^{\delta_{\Gamma}} \equiv \pm 3 \bmod 8$ or $q^{\delta_{\Gamma}} \equiv \pm 1 \bmod 8$. Note that $Q_{\Gamma}$ is unique up to

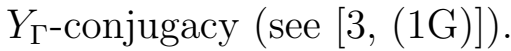

Let $x_{\Gamma} \in Q_{\Gamma}$ such that $\left|x_{\Gamma}\right|=4$ and $x_{\Gamma}$ is primary viewed as an element of $G\left(W_{\Gamma}\right)$, so that $x_{\Gamma}$ is uniquely determined up to conjugacy in $G\left(W_{\Gamma}\right)$. Let $A\left(S_{\Gamma}\right):=Z\left(S_{\Gamma}\right)\left\langle x_{\Gamma}\right\rangle \leq$ $S_{\Gamma}$ and $L_{0}(\Gamma)=C_{G\left(U_{\Gamma}\right)}\left(S_{0}\right)$, so that

$$
L(\Gamma):=C_{G\left(W_{\Gamma}\right)}\left(A\left(S_{\Gamma}\right)\right)=\mathrm{GL}^{\epsilon}\left(\delta_{\Gamma}^{\prime}, q^{e 2^{\alpha_{\Gamma}}}\right) \times \mathrm{GL}^{\epsilon}\left(\delta_{\Gamma}^{\prime}, q^{e 2^{\alpha_{\Gamma}}}\right) \text { or } \mathrm{GL}^{\epsilon}\left(d_{\Gamma}, q^{e_{\Gamma}}\right)
$$

and $L_{0}(\Gamma)=\mathrm{GL}^{\epsilon}\left(\delta_{\Gamma}^{\prime}, q^{e 2^{\alpha_{\Gamma}}}\right)$ or $G\left(U_{\Gamma}\right)$ according as $4 \mid\left(q^{\delta_{\Gamma}}-\epsilon_{\Gamma}\right)$ or $4 \nmid\left(q^{\delta_{\Gamma}}-\epsilon_{\Gamma}\right)$. Let

$$
A(D):=\prod_{\Gamma} A\left(D_{\Gamma}\right), \quad A\left(D_{\Gamma}\right):=S_{0} \times A\left(S_{\Gamma}\right)^{\left(m_{\Gamma}-n_{\Gamma}\right) / 2} \leq E\left(D_{\Gamma}\right) .
$$

Then $A(D)$ is abelian and $A(D)=D$ if and only if $D$ is abelian. In addition,

$$
C_{G}(A(D))=\prod_{\Gamma}\left(L_{0}(\Gamma)^{n_{\Gamma}} \times L(\Gamma)^{\left(m_{\Gamma}-n_{\Gamma}\right) / 2}\right),
$$

where $L(\Gamma)$ is given by $(6.8)$ and $L_{0}(\Gamma)^{n_{\Gamma}}:=1$ when $n_{\Gamma}=0$. Thus $C_{G}(A(D))$ is regular in $G, s \in C_{G}(A(D))$ and

$$
C_{C_{G}(A(D))}(s)=\prod_{\Gamma}\left(\mathrm{GL}^{\epsilon_{\Gamma}}\left(1, q^{\delta_{\Gamma}}\right)^{n_{\Gamma}} \times \mathrm{GL}^{\epsilon_{\Gamma}}\left(1, q^{e_{\Gamma} \delta_{\Gamma}}\right)^{\left(m_{\Gamma}-n_{\Gamma}\right) / e_{\Gamma}}\right) .
$$

Let $(A(D), b)$ be a $B$-subgroup, so that $b=\mathcal{E}_{2}\left(C_{G}(A(D)),(s)\right)$ and $D(b)$ is a Sylow 2-subgroup of $C_{C_{G}(A(D))}(s)$. But $C_{C_{G}(A(D))}(s)$ is a maximal torus, so $D(b)=$ $\mathrm{O}_{2}\left(C_{C_{G}(A(D))}(s)\right)$ is abelian. 
Suppose $m_{\Delta} \geq 2$ for some $\Delta$. By (6.7), there exists $u_{\Delta} \in N_{H_{\Delta}}\left(Q_{\Delta}\right)$ with $\left|u_{\Delta}\right|=3$ and three elements $x_{\Delta, 1}, x_{\Delta, 2}, x_{\Delta, 3}$ of $Q_{\Delta}$ such that $\left|x_{\Delta, i}\right|=4$ and $u_{\Delta}$ acts transitively on the set $\left\{x_{\Delta, 1}, x_{\Delta, 2}, x_{\Delta, 3}\right\}, Q_{\Delta}$ is generated by any two distinct elements in $\left\{x_{\Delta, 1}, x_{\Delta, 2}, x_{\Delta, 3}\right\}$ and $\left[u_{\Delta}, Z\left(S_{\Delta}\right)\right]=1$.

Let $y_{\Delta} \in G\left(V_{\Delta}\right)$ such that

$$
\left[V_{\Delta}, y_{\Delta}\right]=\left[V_{\Delta}, H_{\Delta}\right]=W_{\Delta}, \quad \text { and }\left.\quad\left(y_{\Delta}\right)\right|_{\left[V_{\Delta}, y_{\Delta}\right]}=u_{\Delta}
$$

and set $y_{\Gamma}=1$ when $\Gamma \neq \Delta$. Define $A_{i}\left(S_{\Delta}\right):=Z\left(S_{\Delta}\right)\left\langle x_{\Delta, i}\right\rangle \leq Z\left(S_{\Delta}\right) Q_{\Delta}, A_{i}\left(D_{\Delta}\right):=$ $S_{0} \times A\left(S_{\Delta}\right)^{\left(m_{\Delta}-n_{\Delta}\right) / 2-1} \times A_{i}\left(S_{\Delta}\right) \leq E\left(D_{\Delta}\right)$, and

$$
A_{i}(D):=\prod_{\Gamma \neq \Delta} A\left(D_{\Gamma}\right) \times A_{i}\left(D_{\Delta}\right), \quad y:=\prod_{\Gamma} y_{\Gamma} .
$$

Then $y \in C_{G}(s),|y|=3$, det $y=1$ and $y$ permutes transitively the three abelian subgroups $\left\{A_{1}(D), A_{2}(D), A_{3}(D)\right\}$. Note that $C_{G}\left(A_{i}(D)\right)$ is given by $(6.9)$,

$$
y=1_{C_{V}(y)} \times u_{\Delta}, \quad[V, y]=W_{\Delta}, \quad A_{i}\left(S_{\Delta}\right) \leq Z\left(S_{\Delta}\right) Q_{\Delta} \leq Z\left(S_{\Delta}\right) H_{\Delta} \leq Y_{\Delta} \leq G\left(W_{\Delta}\right) .
$$

Let $Q\left(D_{\Delta}\right)=\left\langle A_{1}\left(D_{\Delta}\right), A_{2}\left(D_{\Delta}\right)\right\rangle$ and $Q(D)=\left\langle A_{1}(D), A_{2}(D)\right\rangle$, so that

$$
Q(D)=\prod_{\Gamma \neq \Delta} A\left(D_{\Gamma}\right) \times Q\left(D_{\Delta}\right), Q\left(D_{\Delta}\right)=S_{0} \times A\left(S_{\Delta}\right)^{\left(m_{\Delta}-n_{\Delta}\right) / 2-1} \times Z\left(S_{\Delta}\right) Q_{\Delta} .
$$

Thus $C_{G\left(V_{\Delta}\right)}\left(Q\left(D_{\Delta}\right)\right)=L_{0}(\Delta)^{n_{\Delta}} \times L(\Delta)^{\left(m_{\Delta}-n_{\Delta}\right) / 2-1} \times C_{G\left(W_{\Delta}\right)}\left(Z\left(S_{\Delta}\right) Q_{\Delta}\right)$,

$$
C_{G\left(W_{\Delta}\right)}\left(Z\left(S_{\Delta}\right) Q_{\Delta}\right)=\mathrm{GL}^{\epsilon}\left(\delta_{\Delta}^{\prime}, q^{e 2^{\alpha} \Delta}\right) \otimes I_{2}:=\left\{x \otimes I_{2}: x \in \mathrm{GL}^{\epsilon}\left(\delta_{\Delta}^{\prime}, q^{e 2^{\alpha} \Delta}\right)\right\},
$$

and

$$
C_{G}(Q(D))=\prod_{\Gamma \neq \Delta}\left(L_{0}(\Gamma)^{n_{\Gamma}} \times L(\Gamma)^{\left(m_{\Gamma}-n_{\Gamma}\right) / 2}\right) \times C_{G\left(V_{\Delta}\right)}\left(Q\left(D_{\Delta}\right)\right) .
$$

Since $A_{1}(D) \leq Q(D) \leq D \leq C_{G}(s)$ and since $C_{G}\left(A_{1}(D)\right) \cap C_{G}(s)$ is a torus, it follows that $C_{C_{G}(Q(D))}(s)$ is a torus.

Let $\left(Q(D), b_{Q}\right)$ be a $B$-subgroup, so that we may suppose $D\left(b_{Q}\right) \leq C_{C_{G}(Q(D))}(s)$. Thus $D\left(b_{Q}\right)$ is abelian and $D\left(b_{Q}\right)=O_{2}\left(C_{C_{G}(Q(D))}(s)\right)$.

If we identify $C_{G\left(W_{\Delta}\right)}\left(Z\left(S_{\Delta}\right) Q_{\Delta}\right)$ with $\operatorname{GL}^{\epsilon}\left(\delta_{\Delta}^{\prime}, q^{e 2^{\alpha} \Delta}\right)$, then by $(6.12)$,

$$
\left\langle Z\left(S_{\Delta}\right) Q_{\Delta}, u_{\Delta}\right\rangle \leq C_{G\left(W_{\Delta}\right)}\left(C_{G\left(W_{\Delta}\right)}\left(Z\left(S_{\Delta}\right) Q_{\Delta}\right)\right) \cong \mathrm{GL}_{2}(q) Z\left(\mathrm{GL}^{\epsilon}\left(\delta_{\Delta}^{\prime}, q^{e 2^{\alpha} \Delta}\right)\right),
$$

since $\mathrm{GL}_{2}(q) \otimes \mathrm{GL}^{\eta}\left(d_{\Delta}, q\right) \leq G\left(W_{\Delta}\right)$ and $\mathrm{GL}^{\epsilon}\left(\delta_{\Delta}^{\prime}, q^{e 2^{\alpha} \Delta}\right) \leq G\left(U_{\Delta}\right)=\mathrm{GL}^{\eta}\left(d_{\Delta}, q\right)$. Thus

$$
\langle Q(D), y\rangle \leq C_{G}\left(C_{G}(Q(D))\right)
$$

and in particular, $y$ centralizes $C_{G}(Q(D))$, and so $b_{Q}^{y}=b_{Q}$. Thus if $B$ is nilpotent, then $m_{\Gamma}(s) \leq 1$ for all $\Gamma \in \mathcal{F}_{q}$.

Conversely, if $m_{\Gamma}(s) \leq 1$ for all $\Gamma \in \mathcal{F}_{q}$, then $C_{\Gamma}=\mathrm{GL}^{\epsilon_{\Gamma}}\left(1, q^{\delta_{\Gamma}}\right)$ and so $N_{C_{\Gamma}}\left(D_{\Gamma}\right)=$ $C_{C_{\Gamma}}\left(D_{\Gamma}\right)=C_{\Gamma}$. Thus $D$ is abelian,

$$
N_{C_{G}(s)}(D)=C_{C_{G}(s)}(D)=C_{G}(s) .
$$


The canonical character of $b_{D}$ is labelled by $(s, 1)$ and is stable in $N_{G}\left(D, b_{D}\right)$. Let $x \in$ $N_{G}\left(D, b_{D}\right)$. Then $s^{x}$ and $s$ are $C_{G}(D)$-conjugate elements of the abelian group $C_{G}(D)$, and so $s^{x}=s$. Hence $x \in C_{G}(s) \leq C_{G}(D)$, and we have shown $N_{G}\left(D, b_{D}\right)=C_{G}(D)$. By Proposition 2.1, $B$ is nilpotent.

The equivalence of (b) and (c) follows by [9].

Recall that for integers $c, m$, we write $2^{c} \| m$ when $2^{c} \mid m$ and $2^{c+1} \nmid m$, and we let $a \geq 2$ be the integer such that $2^{a+1} \|\left(q^{2}-1\right)$.

Remark 6.2 (a) Suppose $G=\mathrm{GL}^{\eta}\left(2 d_{\Delta}, q\right)=G\left(W_{\Delta}\right)=G(V)$ with $2 \mid d_{\Delta}$. Following the notation in the proof above, let $Q^{\prime}(D)=Q_{\Delta} \leq S\left(W_{\Delta}\right) \cap D$ and $A_{i}^{\prime}(D)=\left\langle x_{\Delta, i}\right\rangle \leq$ $Q_{\Delta}$. Then $Q^{\prime}(D) \leq S(V)$,

$$
C_{G}\left(A_{i}^{\prime}(D)\right)=\mathrm{GL}^{\eta}\left(d_{\Delta}, q\right) \times \mathrm{GL}^{\eta}\left(d_{\Delta}, q\right) \quad \text { or } \quad \mathrm{GL}^{\epsilon}\left(d_{\Delta}, q^{e}\right)
$$

according as $4 \mid(q-\eta)$ or $2 \|(q-\eta)$, and $C_{G}\left(Q^{\prime}(D)\right)=\mathrm{GL}^{\epsilon}\left(d_{\Delta}, q\right) \otimes I_{2}$. In addition, if $\left(Q^{\prime}(D), b_{Q^{\prime}}\right)$ is a B-subgroup, then $D\left(b_{Q^{\prime}}\right)$ is abelian, y centralizes $C_{G}\left(Q^{\prime}(D)\right)$ and so $b_{Q^{\prime}}^{y}=b_{Q^{\prime}}$.

(b) Suppose $m_{\Delta} \geq 2$ and follow the notation in the proof above. Then

$$
V=V_{1} \perp V_{2}, \quad D=D_{1} \times D_{2},
$$

where $D_{1}$ is abelian and each direct component of $D_{2}$ is a wreath product of the form $S_{\Gamma} \prec X_{2^{c}}, V_{1}=\left[D_{1}, V\right], V_{2}=\left[D_{2}, V\right]$. Thus

$$
Q(D)=D_{1} \times Q\left(D_{2}\right), \quad Q\left(D_{2}\right)=Q_{0}\left(D_{2}\right) \times Q_{1}\left(D_{2}\right), \quad Q_{1}\left(D_{2}\right)=Z\left(S_{\Delta}\right) Q_{\Delta},
$$

where each direct component of $Q_{0}\left(D_{2}\right)$ has the form $Z\left(S_{\Gamma}\right)\left\langle x_{\Gamma}\right\rangle$. Let $Q=Q(D)$ or $Z(Q(D)), Q_{2}=Q\left(D_{2}\right)$ or $Z\left(Q\left(D_{2}\right)\right)$ and $R=Q \cap S(V)$. Then $C_{G}(R)=C_{G}(Q)$ except when the rank of $\Omega_{1}\left(D_{1}\right)$ is 2 and $D_{1} \cap S\left(V_{1}\right)=\left\{ \pm 1_{V_{1}}\right\}$, in which case,

$$
C_{G}(R)=G\left(V_{1}\right) \times C_{G\left(V_{2}\right)}\left(Q_{2}\right) .
$$

In particular, if $\left(R, b_{R}\right) \leq\left(Q, g_{Q}\right) \leq\left(Q(D), b_{Q}\right)$, then $D\left(b_{R}\right)=D\left(g_{Q}\right)$ and $b_{R}^{y}=b_{R}$ as $y \in S\left(V_{2}\right)$ and $y$ centralizes $C_{G\left(V_{2}\right)}\left(Q\left(D_{2}\right)\right)$.

(c) Following the notation of $(b)$, let $P=Z(Q(D))$, so that $C_{G}(P)$ is regular and $y \in C_{G}(P) \cap S(V)$. If $Z_{G}:=O_{2}\left(C_{G}(P)\right)$ and $\left(P, b_{P}\right) \leq\left(Q(D), b_{Q}\right)$, then $D\left(b_{P}\right) / Z_{G} \cong$ $D_{2^{c+1}}$ with $c \geq a, Z_{G} \leq Z\left(C_{G}(P)\right)$, and $y Z_{G}$ normalizes $\left(Q(D) Z_{G}\right) / Z_{G} \cong \mathbb{Z}_{2} \times \mathbb{Z}_{2}$.

Proof of $(a)$ Since $2 \mid d_{\Delta}$, it follows that $\epsilon_{\Delta}=1$ and $D=\mathbb{Z}_{2^{a+1+\alpha}}\left\langle\mathbb{Z}_{2}\right.$ with $2^{a+1+\alpha} \|\left(q^{d_{\Delta}}-\right.$ $1)$. Since $\operatorname{SL}\left(2, q^{e 2^{\alpha} \Delta}\right)$ contains subgroups $\mathbb{Z}_{q^{e 2^{\alpha} \Delta_{ \pm}}}$, it follows that $D \leq S(V)$. The rest of (a) is clear.

Proofs of $(b)$ and $(c)$ If $4 \mid\left(q^{\delta_{\Gamma}}-\epsilon_{\Gamma}\right)$, then $S_{\Gamma}=\mathbb{Z}_{2^{c}} \succ \mathbb{Z}_{2}$ and $Z\left(S_{\Gamma}\right)=O_{2}\left(\mathrm{GL}^{\epsilon_{\Gamma}}\left(1, q^{\delta_{\Gamma}}\right)\right)$, where $c \geq a \geq 2$. If $4 \nmid\left(q^{\delta_{\Gamma}}-\epsilon_{\Gamma}\right)$, then $S_{\Gamma}$ is a semidihedral group $S D_{2^{a+2}}$ and $Z\left(S_{\Gamma}\right)=\left\{ \pm 1_{W_{\Gamma}}\right\}$. So $C_{G\left(V_{2}\right)}\left(R_{2}\right)=C_{G\left(V_{2}\right)}\left(Q_{2}\right)$ with $R_{2}=Q_{2} \cap S\left(V_{2}\right)$ and the rest of (b) is clear. 
Let $\eta_{\Gamma}=0$ or 2 according as $\Gamma \neq \Delta$ or $\Gamma=\Delta$. Then $P=D_{1} \times Q_{0}\left(D_{2}\right) \times Z\left(S_{\Delta}\right)$,

$$
C_{G}(P)=\prod_{\Gamma}\left(L_{0}(\Gamma)^{n_{\Gamma}} \times L(\Gamma)^{\left(m_{\Gamma}-n_{\Gamma}\right) / 2-\eta_{\Gamma}}\right) \times \mathrm{GL}^{\epsilon}\left(2 \delta_{\Delta}^{\prime}, q^{e 2^{\alpha} \Delta}\right) .
$$

Let $M(H)=C_{H}([H, H])$ for any $H \leq G$. Then $M\left(S_{\Gamma}\right) \cong \mathbb{Z}_{2^{c}} \times \mathbb{Z}_{2^{c}}$ or $\mathbb{Z}_{2^{a+1}}$ according as $4 \mid\left(q^{\delta_{\Gamma}}-\epsilon_{\Gamma}\right)$ or $4 \nmid\left(q^{\delta_{\Gamma}}-\epsilon_{\Gamma}\right)$, and in the former case, $M\left(S_{\Gamma}\right)$ is the base subgroup of $S_{\Gamma}$. In both cases, $C_{G\left(W_{\Gamma}\right)}\left(M\left(S_{\Gamma}\right)\right)=L(\Gamma)$ and $O_{2}(L(\Gamma))=M\left(S_{\Gamma}\right)$. Thus

$$
O_{2}\left(C_{G}(P)\right)=\prod_{\Gamma}\left(O_{2}\left(L_{0}(\Gamma)^{n_{\Gamma}}\right) \times M\left(S_{\Gamma}\right)^{\left(m_{\Gamma}-n_{\Gamma}\right) / 2-\eta_{\Gamma}}\right) \times Z\left(S_{\Delta}\right),
$$

$D\left(b_{P}\right)=O_{2}\left(C_{C_{G}(P)}(s)\right)=\prod_{\Gamma}\left(O_{2}\left(L_{0}(\Gamma)\right)^{n_{\Gamma}} \times M\left(S_{\Gamma}\right)^{\left(m_{\Gamma}-n_{\Gamma}\right) / 2-\eta_{\Gamma}}\right) \times S_{\Delta}$ and $O_{2}\left(C_{G}(P)\right) \leq$ $Z\left(C_{G}(P)\right)$. Thus $D\left(b_{P}\right) / Z_{G} \cong S_{\Delta} / Z\left(S_{\Delta}\right) \cong D_{2^{c+1}}$ or $D_{2^{a+1}}$. Since $y \in N_{C_{G}(P)}(Q(D))$ and $Z_{G} \leq Z\left(C_{G}(P)\right)$, it follows that $y Z_{G}$ normalizes $\left(Q(D) Z_{G}\right) / Z_{G}$.

\section{$7 \quad$ A condition related to nilpotency}

In order to investigate nilpotent blocks of exceptional groups of Lie type using the classical groups, it is not sufficient just to find the nilpotent blocks of classical groups. We need in addition to specify how exactly blocks of classical groups with nonabelian defect groups fail to be nilpotent. To do so we introduce the following condition. In particular it specifies an element of order three causing the block to be non-nilpotent, and relates blocks with those of covering groups. We have in mind groups of Lie type and extensions by diagonal automorphisms.

Property 7.1 Let $K$ be a normal subgroup of a finite group $H$ and $p=2$, and let $B \in \operatorname{Blk}(K)$ and $B_{H} \in \operatorname{Blk}(H)$ such that $B_{H}$ covers $B$.

(a) There exist $B$-subgroups $(P, g) \leq(R, b)$ and an element $y \in N_{C_{K}(P)}(R) \backslash C_{K}(R)$ such that $P \leq Z(R), \ell(g) \geq 2, D(b)$ is abelian,

$$
|y|=3 \quad \text { and } \quad b^{y}=b .
$$

Moreover, if $\left(R, b_{H}\right)$ is a $B_{H}$-subgroup with $b_{H}$ covering $b$, then $D\left(b_{H}\right)$ is abelian and $b_{H}^{y}=b_{H}$.

(b) Both $D(B)$ and $D\left(B_{H}\right)$ are abelian.

Proposition 7.2 Suppose one of Property $7.1(a)$ or $(b)$ holds for a block B. Then B is nilpotent if and only if $l\left(b_{Q}\right)=1$ for every $B$-subgroup $\left(Q, b_{Q}\right)$.

Proof: If (a) holds, then the result is immediate since $l(g)>1$. If (b) holds, then this is [27, Theorem 3].

We prove a lemma which will be useful in establishing the given properties. Let $H$ be a finite group, $K \triangleleft H, Z \leq Z(H) \cap K$ and $\bar{K}:=K / Z \leq \bar{H}:=H / Z$. Let $\bar{B} \in \operatorname{Blk}(\bar{K})$ 
and $B \in \operatorname{Blk}(K)$ dominating $\bar{B}$, and $\left(Q, b_{Q}\right)$ a $B$-subgroup. Let $\gamma: H \rightarrow \bar{H}$ be the natural homomorphism and $\bar{X}=\gamma(X)$ for any $X \subseteq H$,

If $Z$ is a $p^{\prime}$-group, then $\left(\bar{Q}, \bar{b}_{Q}\right)$ is defined in Section 2 and it is a $\bar{B}$-subgroup. Suppose $Z$ is a $p$-group. Then $\gamma^{-1}\left(C_{\bar{K}}(\bar{Q})\right) \leq N_{K}(Q Z)$ and $\gamma^{-1}\left(C_{\bar{K}}(\bar{Q})\right) / C_{K}(Q)$ is a $p$-group. Thus $\gamma^{-1}\left(C_{\bar{K}}(\bar{Q})\right)$ has a unique block $\hat{b}_{Q}$ covering $b_{Q}$ and we denote $\bar{b}_{Q}$ the block of $C_{\bar{K}}(\bar{Q})$ corresponding to $\hat{b}_{Q}$, so that by [31, Lemma 1$],\left(\bar{Q}, \bar{b}_{Q}\right)$ is also a $\bar{B}$-subgroup.

In general, since $\bar{K} \cong\left(K / O_{p}(Z)\right) /\left(Z / O_{p}(Z)\right)$ and $Z / O_{p}(Z) \leq Z\left(K / O_{p}(Z)\right)$, it follows that $\left(\bar{Q}, \bar{b}_{Q}\right)$ is defined and is a $\bar{B}$-subgroup.

Lemma 7.3 Let $H$ be a finite group, $K \triangleleft H, Z \leq Z(H) \cap K$. Define $\bar{K}:=K / Z$ and $\bar{H}:=H / Z$. Let $\bar{B} \in \operatorname{Blk}(\bar{K})$ and $B \in \operatorname{Blk}(K)$ dominating $\bar{B}$. Suppose B-subgroups $(P, g) \leq(R, b)$ satisfy Property $7.1(a)$. In addition, if $Z=O_{2}(Z)$, then suppose, moreover that $C_{H}(P) / Z=C_{\bar{H}}(\bar{P})$ and $C_{H}(R) R / Z=C_{\bar{H}}(\bar{R})$. Then the $\bar{B}$-subgroups $(\bar{P}, \bar{g}) \leq(\bar{R}, \bar{b})$ satisfy Property $7.1(a)$.

Proof: Let $\bar{B}_{\bar{H}} \in \operatorname{Blk}(\bar{H})$ covering $\bar{B}$, and $B_{H} \in \operatorname{Blk}(H)$ dominating $\bar{B}_{\bar{H}}$ and $\chi \in \operatorname{Irr}\left(\bar{B}_{\bar{H}}\right)$, so that $\chi$ covers some $\psi \in \operatorname{Irr}(\bar{B})$. But $\operatorname{Irr}\left(\bar{B}_{\bar{H}}\right) \subseteq \operatorname{Irr}\left(B_{H}\right)$ and $\operatorname{Irr}(\bar{B}) \subseteq$ $\operatorname{Irr}(B)$, so $B_{H}$ covers $B$.

Let $f$ be the unique block of $Z$ covered by $B$. Then each character $\chi$ of $\operatorname{Irr}(B)$ covers a character of $\operatorname{Irr}(f)$. Since $\operatorname{Irr}(\bar{B}) \subseteq \operatorname{Irr}(B)$, it follows that $f$ is principal. Since $(P, g)$ is a $B$-subgroup and $Z \leq Z(K)$ and since $B$ covers $f$, it follows that $g$ covers $f$, and similarly, $b$ covers $f$.

Since $C_{K}(P Z)=C_{K}(P)$, it follows that we may suppose $O_{2}(Z) \leq P$. Let $y \in$ $N_{C_{K}(P)}(R) \backslash C_{K}(R)$ with $y^{3}=1$ and $b^{y}=b$.

We may suppose $Z$ is either a 2-group or a $2^{\prime}$-group. Then by [31, Lemma 1 (iii)] and Lemma 2.6, $(\bar{P}, \bar{g}) \leq(\bar{R}, \bar{b})$ are $\bar{B}$-subgroups. If $Z$ is a $2^{\prime}$-group, then $C_{K}(R) / Z=$ $C_{\bar{K}}(\bar{R})$. If $Z$ is a 2-group, then $C_{H}(R) / Z=C_{\bar{H}}(\bar{R}), \gamma^{-1}\left(C_{\bar{H}}(\bar{R})\right)=C_{H}(R)$ and so $\gamma^{-1}\left(C_{\bar{K}}(\bar{R})\right)=C_{K}(R)$. Thus $C_{K}(R) / Z=C_{\bar{K}}(\bar{R})$ and $D(b) / Z=D(\bar{b})$. So $D(\bar{b})$ is abelian as $D(b)$ is abelian.

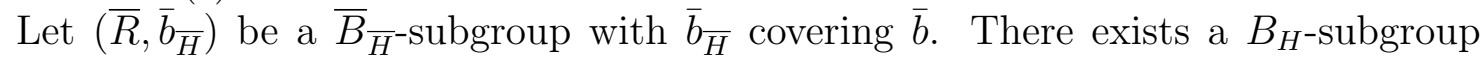
$\left(R, b_{H}\right)$ such that $b_{H}$ dominates $\bar{b}_{\bar{H}}$. Then $b_{H}$ covers $b$ and so $b_{H}$ has an abelian defect group $D\left(b_{H}\right)$ and hence $D\left(\bar{b}_{\bar{H}}\right)=D\left(b_{H}\right) Z / Z$ is abelian.

Since $|y|=3$ and $y \in N_{C_{K}(P)}(R) \backslash C_{K}(R)$, it follows that $|\bar{y}|=3$ and $\bar{y} \in$ $N_{C_{\bar{K}}(\bar{P})}(\bar{R}) \backslash C_{\bar{K}}(\bar{R})$. Since the canonical character $\theta_{H}$ of $b_{H}$ is the lift of the canonical character $\bar{\theta}_{H}$ of $\bar{b}_{H}$ and since $\theta_{H}^{y}=\theta_{H}$, it follows that $\bar{b}_{H}^{\bar{y}}=\bar{b}_{H}$. Similarly, $\bar{b}^{\bar{y}}=\bar{b}$ and since $\ell(\bar{g})=\ell(g)$, it follows that $\ell(\bar{g}) \geq 2$.

\section{Classical groups}

Proposition 8.1 Let $K:=\mathrm{SL}_{n}^{\eta}(q) \leq H \leq G:=\mathrm{GL}_{n}^{\eta}(q)=\mathrm{GL}^{\eta}(V), Z \leq Z(K)$, $B \in \operatorname{Blk}(K), B_{H} \in \operatorname{Blk}(H)$ covering $B$. Let $B_{G} \in \operatorname{Blk}(G)$ be a weakly regular cover of $B_{H}$, and $R:=Q\left(D\left(B_{G}\right)\right) \cap K$, except when $D\left(B_{G}\right)=\mathbb{Z}_{2^{a+1+\alpha}}\left\{\mathbb{Z}_{2}\right.$ for some $\alpha \geq 0$, in which case $R=Q^{\prime}(D)$ as defined in Remark $6.2(a)$. Then either Property $7.1(a)$ holds 
for $(Z(R), g) \leq(R, b)$ with $C_{H}(Z(R)) / Z=C_{\bar{H}}(Z(R) / Z)$ and $C_{H}(R) R / Z=C_{\bar{H}}(\bar{R})$, or Property 7.1 (b) holds.

Proof: Suppose $B_{G}=\mathcal{E}_{2}(G,(s))$. Since $B_{H}$ covers $B$, it follows that $D(B)=$ $D\left(B_{H}\right) \cap K$ for some defect group $D\left(B_{H}\right)$. There exists a defect group $D\left(B_{G}\right)$ such that $D\left(B_{H}\right)=D\left(B_{G}\right) \cap H$, so

$$
D(B)=D\left(B_{H}\right) \cap K=D\left(B_{G}\right) \cap K \quad \text { and } \quad D\left(B_{H}\right)=D\left(B_{G}\right) \cap H .
$$

We may suppose $D\left(B_{G}\right) \in \operatorname{Syl}_{2}\left(C_{G}(s)\right)$.

Set $R_{G}=Q\left(D\left(B_{G}\right)\right)$ or $Q^{\prime}\left(D\left(B_{G}\right)\right)$ according as $D\left(B_{G}\right) \nsucceq \mathbb{Z}_{2^{a+1+\alpha}} \prec \mathbb{Z}_{2}$ for all $\alpha \geq 0$ or $D\left(B_{G}\right) \cong \mathbb{Z}_{2^{a+1+\alpha}}\left\{\mathbb{Z}_{2}\right.$ for some $\alpha \geq 0$. Then $R_{G}$ and $C_{G}\left(R_{G}\right)$ are given by (6.13) or Remark 6.2 (a) with $Q(D)$ or $Q^{\prime}(D)$ replaced by $R_{G}$.

In the notation of the proof of Theorem 6.1 , suppose $m_{\Gamma} \leq 1$ for all $\Gamma$. Then $D\left(B_{G}\right)$ is abelian, and both $D(B)$ and $D\left(B_{H}\right)$ are abelian. So we suppose, moreover $m_{\Delta} \geq 2$ for some $\Delta$. There exists $y \in C_{G}(s) \cap K$ such that $y \in N_{G}\left(R_{G}\right) \backslash C_{G}\left(R_{G}\right),|y|=3$, $\left.y\right|_{V_{\Gamma}}=1_{V_{\Gamma}}$ for all $\Gamma \neq \Delta$. Let $R_{H}:=R_{G} \cap H$ and $R=R_{G} \cap K$.

(1). Note that $C_{G}(R)$ is given in Remark 6.2 (b). Let $(R, b)$ be a $B$-subgroup, $\left(R, b_{H}\right)$ a $B_{H}$-subgroup such that $b_{H}$ covers $b$, and $\left(R, b_{G}\right)$ a $B_{G}$-subgroup such that $b_{G}$ coves $b_{H}$. By Remark $6.2(\mathrm{~b})$ and the proof of Theorem 6.1, $D\left(b_{G}\right)$ is abelian, so both $D(b)$ and $D\left(b_{H}\right)$ are abelian as we may suppose $D(b)=D\left(b_{H}\right) \cap C_{K}(R)$ and $D\left(b_{H}\right)=D\left(b_{G}\right) \cap C_{H}(R)$.

Let $P=Z(R),(P, g) \leq(R, b),\left(P, g_{G}\right) \leq\left(R, b_{G}\right)$ such that $g_{G}$ covers $g$ and $y \in$ $N_{C_{K}(P)}\left(R, b_{G}\right) \backslash C_{G}(R)$ given by (6.11). By (6.14), $y$ centralizes $C_{G}(R)$, so $b^{y}=b$ and hence $y \in N_{C_{K}(P)}(R, b)$ as $C_{K}(R) \leq C_{G}(R)$. Similarly, $y \in N_{C_{H}(P)}\left(R, b_{H}\right)$.

Suppose $m_{\Delta}=2$ and $m_{\Gamma}=0$ for all $\Gamma \neq \Delta$. Then $R=O_{2}(Z(K)) Q_{8}$ or $Q_{8}$ according as $d_{\Delta}$ is odd or even. Thus $G=G^{\eta}\left(2 d_{\Delta}, q\right), P=Z(R) \leq Z(G)$ and so $C_{G}(P)=G$. Suppose $x \in G$ such that for any $u \in R, x^{-1} u x=z u$ for some $z \in O_{2}(Z(G))$, so that $x \in N_{G}(R)$. Since $\operatorname{Out}\left(Q_{8}\right)=O_{2}^{-}(2)=\mathrm{GL}_{2}(2)$, it follows that if $x \notin C_{G}(R) R$, then $x^{-1} y_{1} x=y_{1}$ and $x^{-1} y_{2} x=y_{1} y_{2}$ for some $y_{i} \in R$ with $\left\langle y_{1}, y_{2}\right\rangle \cong Q_{8}$. Now $z y_{2}=x^{-1} y_{2} x=y_{1} y_{2}$ and $y_{1}=z \in Z(G)$, which is impossible. It follows that $x \in C_{G}(R) R$ and so $C_{G}(R) R / Z=C_{G / Z}(R / Z)$.

Suppose $m_{\Delta} \geq 3$ or $m_{\Delta}=2$ but $m_{\Gamma} \geq 1$ for some $\Gamma \neq \Delta$. A similar proof to above shows that for any $1 \neq Z \leq Z(K), C_{G}(P) / Z=C_{G / Z}(P / Z)$ and $C_{G}(R) R / Z=$ $C_{G / Z}(R / Z)$.

(2). Suppose $G=\mathrm{GL}^{\eta}\left(2 d_{\Delta}, q\right)$ and $D\left(B_{G}\right)=\mathbb{Z}_{2^{a+1+\alpha}} \backslash \mathbb{Z}_{2}$ with $\alpha \geq 0$ for some $\Delta$. By Remark $6.2(\mathrm{a}), D(B)=D\left(B_{G}\right), R=Q_{8}, P \leq Z(K)$ and $g=B$. But $Q(D(B))=Z(D(B)) Q_{8}$ and $y \in N_{K}\left(Q(D), b_{Q}\right)$, so by Lemma $2.4, \ell(B) \geq 2$.

Suppose $D\left(B_{G}\right) \neq \mathbb{Z}_{2^{a+1+\alpha}} \backslash \mathbb{Z}_{2}$, so that $R_{G}=Q\left(D\left(B_{G}\right)\right)$. Let $P_{G}=Z\left(R_{G}\right)$, $Z_{G}=O_{2}\left(C_{G}\left(P_{G}\right)\right)$ and $Z_{K}=Z_{G} \cap K$. By Remark $6.2(\mathrm{c}), Z_{G} \leq Z\left(C_{G}\left(P_{G}\right)\right)$ and $D\left(g_{G}\right) / Z_{G}=D_{2^{c+1}}$ with $c \geq a$. Since $R \leq D(g)=D\left(g_{G}\right) \cap K$, it follows that $\bar{R}:=R / Z_{K} \leq D(g) / Z_{K} \leq D_{2^{c+1}}$. By Lemma $2.4, \ell(g) \geq 2$ and Property 7.1 (a) holds.

Let $V$ be a non-degenerate orthogonal or symplectic space, $G=I_{0}(V)$ and let $G^{*}$ be the dual group of $G$. Then

$$
\mathrm{Sp}_{2 n}(q)^{*}=\mathrm{SO}_{2 n+1}(q), \quad \mathrm{SO}_{2 n+1}(q)^{*}=\mathrm{Sp}_{2 n}(q), \quad \mathrm{SO}_{2 n}^{\eta}(q)^{*}=\mathrm{SO}_{2 n}^{\eta}(q)
$$


If $B$ is a 2-block of $I_{0}(V)$, then $B=\mathcal{E}_{2}\left(I_{0}(V),(s)\right)$ for a semisimple 2'-element $s \in$ $I_{0}(V)^{*}$. Let $\left(D, b_{D}\right)$ be a Sylow $B$-subgroup. By $[2,(5.1)], V$ and $D$ have decompositions

$$
V=V_{0} \perp V_{+}, \quad D=D_{0} \times D_{+},
$$

where $D_{0} \leq I_{0}\left(V_{0}\right)$ is an elementary abelian 2-subgroup and $D_{+} \leq I_{0}\left(V_{+}\right)$. Let $G_{0}:=$ $I_{0}\left(V_{0}\right), G_{+}:=I_{0}\left(V_{+}\right), C_{+}:=C_{I_{0}\left(V_{+}\right)}\left(D_{+}\right)$and let $V^{*}$ be the underlying space of $I_{0}(V)^{*}$.

Let $z \in D$ be a primitive element. Then $z$ is given by [2, Remark 2.2 .9 (2)], so $|z|=4$ and $\left[V, D_{+}\right]=[V, z]=V_{+}$. Thus

$$
z=z_{0} \times z_{+}, \quad L:=C_{G}(z)=L_{0} \times L_{+}, \quad L_{0}=G_{0}, \quad L_{+}:=\operatorname{GL}^{\epsilon}(m, q),
$$

where $z_{0}=1_{V_{0}}, z_{+} \leq D_{+}$and $\operatorname{dim} V_{+}=2 m$. Then $L$ is a regular subgroup of $G$ and we may suppose $s \in L^{*} \leq G^{*}$. In particular,

$$
V^{*}=U_{0} \perp U_{+} \quad \text { and } \quad s=s_{0} \times s_{+},
$$

where $U_{0}=V_{0}^{*}, s_{0} \in L_{0}^{*}=I_{0}\left(U_{0}\right), U_{+}$is the underlying space of $L_{+}^{*}$ and $s_{+} \in L_{+}^{*} \leq$ $I_{0}\left(U_{+}\right)$.

Let $C_{I\left(U_{+}\right)}\left(s_{+}\right)=\prod_{\Gamma} C_{\Gamma}$ and let $U_{\Gamma}$ be the underlying vector space of $C_{\Gamma}$, so that

$$
C_{\Gamma}=\mathrm{GL}^{\epsilon_{\Gamma}}\left(m_{\Gamma}\left(s_{+}\right), q^{\delta_{\Gamma}}\right) \quad \text { or } \quad I\left(U_{\Gamma}\right)
$$

according as $\Gamma \neq X \pm 1$ or $\Gamma=X \pm 1$.

Proposition 8.2 Let $K:=\Omega_{2 n}^{\eta}(q):=\Omega^{\eta}(V) \leq H \leq J_{0}(V), B_{K} \in \operatorname{Blk}(K)$ and $B_{H} \in \operatorname{Blk}(H)$ covering $B_{K}$. Either Property $7.1(a)$ holds for some $B_{K}$-subgroups $(Z(R), g) \leq(R, b)$ with $C_{H}(Z(R)) / Z=C_{H / Z}(Z(R) / Z)$ and $C_{H}(R) R / Z=C_{H / Z}(R / Z)$ for any $Z \leq Z(K)$, or Property 7.1 (b) holds.

Proof: Let $G:=\mathrm{SO}_{2 n}^{\eta}(q):=\mathrm{SO}(V)$ and $B \in \operatorname{Blk}(G)$ covering $B_{K}$. Then $D\left(B_{K}\right)=$ $D \cap K$ for some defect group $D:=D(B)$. Since $G$ is self dual, we have $V=V^{*}, U_{0}=$ $V_{0}, U_{+}=V_{+}$in (8.3).

(1). We may suppose $D \in \operatorname{Syl}_{2}\left(C_{G}(s)\right)$ and so $D\left(B_{K}\right) \in \operatorname{Syl}_{2}\left(C_{K}(s)\right)$. In particular, $D$ is abelian if and only if $m_{\Gamma}(s) \leq 1$ if and only if $C_{G}(s)$ is a maximal torus with $D=O_{2}\left(C_{G}(s)\right)$, if and only if $D\left(B_{K}\right)$ is abelian.

Suppose $m_{\Delta}(s) \geq 2$ for some $\Delta$, so that $m_{\Delta}\left(s_{+}\right) \geq 2$. In the notation given in (8.1), (8.2) and (8.3), we have $D_{0} \in \operatorname{Syl}_{2}\left(C_{G_{0}}\left(s_{0}\right)\right)$ and $D_{+} \in \operatorname{Syl}_{2}\left(C_{G_{+}}\left(s_{+}\right)\right)$. In general, $z \notin K$ and we modify $z$ as follows. Since $L_{+}=\operatorname{GL}^{\epsilon}(m, q)$ and $D_{+} \in \operatorname{Syl}_{2}\left(C_{L_{+}}\left(s_{+}\right)\right)$, it follows that if we view $s_{+}$as an element of $L_{+}$, we still have $m_{\Gamma}\left(s_{+}\right) \geq 2$ for some $\Gamma$, and so by Remark 6.2 (b), $D_{+}=D_{1} \times D_{2}$ given by (6.16). Thus $z_{+}=z_{1} \times z_{2}$ for some $z_{1} \in D_{1}$ and $z_{2} \in D_{2}$. Let $z_{+}^{\prime}:=1_{D_{1}} \times z_{2}$ and $z^{\prime}=z_{0} \times z_{+}^{\prime}$. Then $z_{+}^{\prime} \in D_{+}, z^{\prime} \in K$ and $z^{\prime} \in D_{K}=D \cap K \in \operatorname{Syl}_{2}\left(C_{K}(s)\right)$.

For simplicity of notation, we write $z=z^{\prime}$, so $V=V_{0} \perp V_{+}$with $V_{0}=C_{V}(z)$, $V_{+}=[V, z], z=z_{0} \times z_{+}, s=s_{0} \times s_{+}$with $s_{0} \in K_{0}$ and $s_{+} \in K_{+}, D=D_{0} \times D_{+}$ with $D_{0} \in \operatorname{Syl}_{2}\left(C_{G_{0}}\left(s_{0}\right)\right)$ and $D_{+} \in \operatorname{Syl}_{2}\left(C_{G_{+}}\left(s_{+}\right)\right)$, where $K_{0}=\Omega\left(V_{0}\right), K_{+}=\Omega\left(V_{+}\right)$, $G_{0}=\mathrm{SO}\left(V_{0}\right)$ and $G_{+}=\mathrm{SO}\left(V_{+}\right)$. In particular, if $\Gamma \neq X \pm 1$, then $m_{\Gamma}\left(s_{+}\right)$is even and if $\Gamma=X \pm 1$, then $4 \mid m_{\Gamma}\left(s_{+}\right)$(cf. the decomposition of (6.16)), and $m_{\Delta}\left(s_{+}\right) \geq 2$. In 
addition, $L=C_{G}(z)=L_{0} \times L_{+}, L_{0}=G_{0}$ and $L_{+}=\mathrm{GL}^{\epsilon}(m, q)$ with $m$ even, and hence $\eta\left(V_{+}\right)=+$and $4 \mid \operatorname{dim} V_{+}$.

Let $\left(z, B_{z}\right)$ be a $B_{K^{-}}$-element and $\left(z, B_{L}\right)$ a $B$-element such that $B_{L}$ covers $B_{z}$. Then $B_{L}=B_{L_{0}} \times B_{L_{+}}$, where $B_{L_{0}} \in \operatorname{Blk}\left(L_{0}\right)$ and $B_{L_{+}} \in \operatorname{Blk}\left(L_{+}\right)$. Thus $B_{L_{0}}=\mathcal{E}_{2}\left(L_{0},\left(s_{0}\right)\right)$ and $B_{L_{+}}=\mathcal{E}_{2}\left(L_{+},\left(s_{+}\right)\right)$. Let $R_{G}=D_{0} \times Q^{\prime \prime}\left(D_{+}\right), R=R_{G} \cap K, R_{0}:=D_{0} \cap \Omega\left(V_{0}\right)$ and $R_{+}:=Q^{\prime \prime}\left(D_{+}\right) \cap \mathrm{SL}^{\epsilon}(m, q) \leq \Omega\left(V_{+}\right)$, where $Q^{\prime \prime}\left(D_{+}\right)=Q^{\prime}\left(D\left(B_{L_{+}}\right)\right)$or $Q\left(D\left(B_{L_{+}}\right)\right)$as in Proposition 8.1. So $z \in R$,

$$
C_{K}(R) \leq C_{G}(R)=C_{L_{0}}\left(R_{0}\right) \times C_{L_{+}}\left(R_{+}\right) \leq L
$$

Let $(R, b)$ be a $B_{z}$-subgroup and $\left(R, b_{G}\right)$ a $B_{L}$-subgroup such that $b_{G}$ covering $b$. Then

$$
b_{G}=b_{0} \times b_{+}, \quad b_{0} \in \operatorname{Blk}\left(C_{L_{0}}\left(R_{0}\right)\right), \quad b_{+} \in \operatorname{Blk}\left(C_{L_{+}}\left(R_{+}\right)\right),
$$

and so $\left(R_{+}, b_{+}\right)$is a $B_{L_{+}}$-subgroup. It follows by Proposition 8.1 that $C_{K}(Z(R)) / Z=$ $C_{K / Z}(Z(R) / Z)$ and $C_{K}(R) R / Z=C_{K / Z}(R / Z)$ for any $Z \leq Z(K)$. By Proposition 8.1 again, $D\left(b_{+}\right)$is abelian, and there exists $y \in \mathrm{SL}^{\epsilon}(m, q) \leq L_{+}$such that $y \in N_{L_{+}}\left(R_{+}\right) \backslash$ $C_{L_{+}}\left(R_{+}\right),|y|=3$ and $b_{+}^{y}=b_{+}$. In particular, $b_{G}^{y}=b_{G}$. Note that $C_{C_{L_{+}}\left(R_{+}\right)}\left(s_{+}\right)$ is a torus of both $L_{+}$and $I_{0}\left(V_{+}\right), D\left(b_{+}\right)=O_{2}\left(C_{C_{L_{+}}\left(R_{+}\right)}\left(s_{+}\right)\right)$, and $C_{C_{L_{+}}\left(R_{+}\right)}\left(s_{+}\right)=$ $C_{C_{G_{+}}\left(R_{+}\right)}\left(s_{+}\right)$.

Since $\left[C_{G}(R): C_{K}(R)\right] \leq 2$ and $|y|=3$, it follows that $b^{y}=b$ and $D(b)=D\left(b_{G}\right) \cap$ $C_{K}(R)$ is abelian. Let $P=Z(R),(P, g) \leq(R, b)$ and $\left(P, g_{G}\right) \leq\left(R, b_{G}\right)$ such that $g_{G}$ covers $g$. Then $g_{G}=g_{0} \times g_{+}$with $g_{0}=b_{0}$ and $\left(Z\left(R_{+}\right), g_{+}\right) \leq\left(R_{+}, b_{+}\right)$. Thus $D\left(g_{+}\right)=\mathbb{Z}_{2^{a+1+\alpha}}\left\{\mathbb{Z}_{2}\right.$ or $D\left(g_{G}\right) / Z_{G} \cong D_{2^{c+1}}$, where $Z_{G}=O_{2}\left(C_{G}(R)\right) \leq Z\left(C_{G}(R)\right)$. If $D\left(g_{+}\right)=\mathbb{Z}_{2^{a+1+\alpha}}\left\{\mathbb{Z}_{2}\right.$, then $D\left(g_{+}\right) \leq \mathrm{SL}^{\epsilon}(m, q) \leq D(b)$ and $D(g) / Z_{K}=D\left(g_{+}\right)$and $Z_{K}:=R_{0} \leq Z\left(C_{K}(P)\right)$. By Lemma $2.4, \ell(g) \geq 2$.

(2). Let $\left(R, b_{H}\right)$ be a $B_{H}$-subgroup such that $b_{H}$ covers $b$. Since $C_{H}(R) / C_{K}(R) Z(H)$ is a 2-group, it follows that $b_{H}^{y}=b_{H}$. In order to show that $D\left(b_{H}\right)$ is abelian and $C_{H}(Z(R)) / Z=C_{H / Z}(Z(R) / Z)$ and $C_{H}(R) R / Z=C_{H / Z}(R / Z)$ for $Z=Z(K)$ we suppose $H=J_{0}(V)$. It follows by [14, (1A)] that

$$
C_{H}(R)=\left\langle C_{L_{0}}\left(R_{0}\right) \times C_{L_{+}}\left(R_{+}\right), \tau\right\rangle, \quad \tau:=\tau_{0} \times \tau_{+},
$$

where $\tau_{0} \in C_{J_{0}\left(V_{0}\right)}\left(R_{0}\right)$ and $\tau_{+} \in C_{J_{0}\left(V_{+}\right)}\left(R_{+}\right)$with $J_{0}(V)=\langle G, \tau\rangle$. Thus $N_{H}(R)=$ $\left\langle N_{G}(R), \tau\right\rangle$. If $x Z \in C_{H / Z}(R / Z)$ for some $x \in H$, then $x \in N_{H}(R)$ and $x^{-1} u x= \pm u$ for any $u \in R$. We may suppose $x=x_{0} \times x_{+} \in C_{L_{0} / Z_{0}}\left(R_{0} / Z_{0}\right) \times C_{L_{+} / Z_{+}}\left(R_{+} / Z_{+}\right)$with $Z_{0}=Z\left(K_{0}\right)$ and $Z_{+}=Z\left(K_{+}\right)$. By Proposition 8.1, $x_{+} \in C_{L_{+}}\left(R_{+}\right)$and so $x \in C_{H}(R)$. Similarly, $C_{H}(Z(R)) / Z=C_{H / Z}(Z(R) / Z)$.

Since $C_{C_{J_{0}\left(V_{0}\right)}\left(R_{0}\right)}\left(s_{0}\right)$ is a maximal torus of $C_{J_{0}\left(V_{0}\right)}\left(R_{0}\right)$ and $C_{C_{J_{0}\left(V_{+}\right)}\left(R_{+}\right)}\left(s_{+}\right)$is a torus of $C_{J_{0}\left(V_{+}\right)}\left(R_{+}\right)$, it follows that $C_{C_{H}(R)}(s)$ is a torus of $C_{H}(R)$. But $D\left(b_{H}\right) \in$ $\operatorname{Syl}_{2}\left(C_{C_{H}(R)}(s)\right)$, so $D\left(b_{H}\right)$ is abelian. This proves Property 7.1 (a).

(3). Suppose $m_{\Gamma}(s) \leq 1$, so that $C_{G}(s)$ is a maximal torus of $G$ and $D=O_{2}\left(C_{G}(s)\right)$ is abelian. By [14, (1B)], $C_{G}(s)$ is a maximal torus of $G$ if and only if $C_{J_{0}(V)}(s)$ is a maximal torus of $J_{0}(V)$. To show that $D\left(B_{H}\right)$ is abelian we may suppose $H=J_{0}(V)$, so that $D\left(B_{H}\right) \in \operatorname{Syl}_{2}\left(C_{J_{0}(V)}(s)\right)$ and hence $D\left(B_{H}\right)$ is abelian. 
Remark 8.3 In the notation of proof $(1)$ above let $A_{+}(i)=A_{i}\left(D_{+}\right) \leq R_{+}$or $A_{i}^{\prime}\left(D_{+}\right) \leq$ $R_{+}$according as $R_{+}=Q\left(D_{+}\right) \cap \mathrm{SL}^{\epsilon}(m, q)$ or $Q^{\prime}\left(D_{+}\right), A(i):=R_{0} \times A_{+}(i)$ for $1 \leq i \leq 3$, where $A_{i}\left(D_{+}\right)$is defined in (6.11) and $A_{i}^{\prime}\left(D_{+}\right)$is given by (6.15). Then $A(i) \leq R, R=$ $\langle A(1), A(2)\rangle$ and $y$ acts transitively on $\{A(1), A(2), A(3)\}$. If $\left(A_{+}(i), b_{A_{+}(i)}\right) \leq\left(R_{+}, b_{+}\right)$, then $b_{A_{+}(i)}=\mathcal{E}_{2}\left(C_{G_{+}}\left(A_{+}(i)\right),\left(s_{+}\right)\right), C_{C_{L_{+}}\left(A_{+}(i)\right)}\left(s_{+}\right)$is a maximal torus of both $L_{+}$and $G_{+}$, and $D\left(b_{A_{+}(i)}\right)=O_{2}\left(C_{C_{L_{+}}\left(A_{+}(i)\right)}\left(s_{+}\right)\right)$. Note also that $C_{G_{+}}\left(R_{+}\right)=C_{L_{+}}\left(R_{+}\right) \leq$ $C_{L_{+}}\left(A_{+}(i)\right), \mathrm{SO}^{\eta}(2, q)=\mathrm{GL}^{\eta}(1, q)$, and we have that

$$
C_{C_{G}(A(i))}(s)=\prod_{\Gamma}\left(\mathrm{GL}^{\epsilon_{\Gamma}}\left(1, q^{\delta_{\Gamma}}\right)^{n_{\Gamma}} \times \mathrm{GL}^{\epsilon_{\Gamma}}\left(1, q^{e_{\Gamma} \delta_{\Gamma}}\right)^{\left(m_{\Gamma}-n_{\Gamma}\right) / e_{\Gamma}}\right)
$$

is a maximal torus of $G$, where $m_{\Gamma}=m_{\Gamma}(s)$ and $n_{\Gamma}=1$ or 0 according as $m_{\Gamma}$ is odd or even.

Proposition 8.4 Let $K:=\Omega_{2 n+1}(q):=\Omega(V)$ or $K:=\operatorname{Sp}_{2 n}(q)=\operatorname{Sp}(V)$, and

$$
K \leq H \leq J_{0}(V),
$$

$B_{K} \in \operatorname{Blk}(K)$ and $B_{H} \in \operatorname{Blk}(H)$ covering $B_{K}$, where $H=\mathrm{SO}(V)$ when $K=$ $\Omega(V)$. Either Property 7.1 (a) holds for some $B_{K}$-subgroups $(Z(R), g) \leq(R, b)$ with $C_{H}(Z(R)) / Z=C_{H / Z}(Z(R) / Z)$ and $C_{H}(R) R / Z=C_{H / Z}(R / Z)$ for any $Z \leq Z(K)$, or Property $7.1(b)$ holds.

Proof: Let $s^{*}$ be a dual element of $s$ in $I_{0}(V)$ given by [3, (4A)]. Replacing $G$ by $H$ and $s$ by $s^{*} \in I_{0}(V)$ in the proof (1) of Proposition 8.2 with some obvious modification, we have that Property 7.1 (a) holds for some $B_{K}$-subgroup $(R, b)$. A proof similar to the proof (3) of Proposition 8.2 with $s$ replaced by $s^{*} \in I_{0}(V)$ shows that Property 7.1 (b) holds.

Theorem 8.5 Let $G=I_{0}(V), B \in \operatorname{Blk}(G)$, and $\left(D, b_{D}\right)$ a Sylow B-subgroup. Follow the notation in (8.1), (8.2) and (8.3). Then $B$ is nilpotent if and only if $C_{I_{0}\left(V^{*}\right)}(s)$ is a maximal torus $T^{*}$ of $I_{0}\left(V^{*}\right)$ and $D=O_{2}(T)$, where $T \leq I_{0}(V)$ is a dual of $T^{*}$.

Proof: Note that $C_{G^{*}}(s)$ is regular and $B=\mathcal{E}_{2}(G,(s))$. By [3, (5A)], we may suppose $D \in \operatorname{Syl}_{2}\left(C_{G}\left(s^{*}\right)\right)$, where $s^{*}$ is a dual element of $s$ in $G$ defined in [3, (4A)]. If $B$ is nilpotent, then by Propositions 8.2 and $8.4, D$ is abelian and so $C_{G}\left(s^{*}\right)$ is a maximal torus of $G$.

Conversely, suppose $T:=C_{G}\left(s^{*}\right)$ is a maximal torus of $G$ and $\theta$ is the canonical character of $b_{D}$. Then $D=O_{2}(T), C_{C_{G}(D)}\left(s^{*}\right)=T$, and $\theta= \pm R_{T}^{C_{G}(D)}(s)$. Thus $N_{G}(D, \theta)=T$ and $B=\mathcal{E}_{2}(G,(s))$ is nilpotent.

Proposition 8.6 Let $K:=\operatorname{Spin}^{\eta}(V) \triangleleft H$ such that $H / K$ is abelian, $C_{H}(K) \leq Z(H)$ and $H / Z(H) \leq J_{0}(V) / Z\left(J_{0}(V)\right)$. Let $B \in \operatorname{Blk}(K), B_{H} \in \operatorname{Blk}(H)$ covering $B$, and $Z \leq$ $Z(K)$ such that $K_{c}:=K / Z=\Omega^{\eta}(V)$, so that $|Z|=\operatorname{gcd}(2, q-\eta)$. Either Property 7.1 (a) holds for some $B$-subgroups $(Z(R), g) \leq(R, b)$ with $C_{H}(Z(R)) / Z=C_{H / Z}(Z(R) / Z)$ and $C_{H}(R) R / Z=C_{H / Z}(R / Z)$ for any $Z \leq Z(K)$, or Property 7.1 (b) holds. 
Proof: We prove the proposition for $K=\operatorname{Spin}_{2 n}^{\eta}(q)=\operatorname{Spin}^{\eta}(V)$. A similar proof with some modifications works for $K=\operatorname{Spin}_{2 n+1}^{\eta}(q)$.

Let $D:=D(B), G:=\mathrm{SO}^{\eta}(V)$ and $Z_{+} \leq Z\left(D_{0}(V)\right)$ such that $G=D_{0}(V) / Z_{+}$, so that $Z=Z_{+} \cap K$ and $Z_{+} \cong \mathbb{Z}_{q-1}$. Identify $G$ with $G^{*}$.

Note that $B$ dominates a unique block $B_{c} \in \operatorname{Blk}\left(K_{c}\right)$ and $B_{c}$ is covered by a unique block $B_{G} \in \operatorname{Blk}(G)$. Thus $B_{G}=\mathcal{E}_{2}(G,(s))$ for some semisimple 2'-element $s \in G$ and $D_{G}:=D\left(B_{G}\right) \in \operatorname{Syl}_{2}\left(C_{G}(s)\right)$. Since $G / K_{c}=\mathbb{Z}_{2}$, it follows that $s \in K_{c}$ and we may suppose $s \in K$, that is, we identify $s \in G=G^{*} \leq J_{0}\left(V^{*}\right)$ with its dual $s^{*} \in K \leq J_{0}\left(V^{*}\right)^{*}=D_{0}(V)$. Note that since $s$ is a $2^{\prime}$-element, it follows that $C_{J_{0}\left(V^{*}\right)}(s)$ is a regular subgroup and so $C_{J_{0}\left(V^{*}\right)}(s)^{*} \cong C_{D_{0}(V)}\left(s^{*}\right)$ for a (unique) semisimple $2^{\prime}$ element $s^{*} \in K \leq D_{0}(V)$, so that it is possible to identify $s$ with $s^{*}$.

Since $B_{G}$ covers $B_{c}$, it follows that $D / Z \in \operatorname{Syl}_{2}\left(C_{K_{c}}(s)\right)$ and by [14, (2E) (2)], $D \in \operatorname{Syl}_{2}\left(C_{K}(s)\right)$. Thus $D_{G}$ is abelian if and only if $m_{\Gamma}(s) \leq 1$ for $\Gamma \neq X-1$ and $m_{X-1}(s) \leq 2$. This happens if and only if $C_{G}(s)$ is a maximal torus of $G$. By [14, $(2 \mathrm{E})$ (2)] again, this happens if and only if $C_{K}(s)$ is a maximal torus of $K$. In particular, $D_{G}$ is abelian if and only if $D$ is abelian.

(1). Suppose $m_{\Delta}(s) \geq 2$ for some $\Delta$. Let $z_{c}$ be a primary element of $D_{G}$ with the modification given by the proof (1) of Proposition 8.2, so that $z_{c} \in D_{G} \cap K_{c}$ and $V=V_{0} \times V_{+}$with $V_{0}=C_{V}\left(z_{c}\right)$ and $V_{+}=\left[V, z_{c}\right]$. Let $z_{c}=z_{0} \times z_{+}, D_{G}=D_{0} \times D_{+}$, $s=s_{0} \times s_{+}$and $L=C_{G}\left(z_{c}\right)=L_{0} \times L_{+}$be the decompositions corresponding to $V=V_{0} \perp V_{+}$, so that $z_{0}=1_{V_{0}}, L_{0}=I_{0}\left(V_{+}\right), L_{+}=C_{G_{+}}\left(z_{+}\right)=\operatorname{GL}^{\epsilon}(m, q)$ with $G_{+}=$ $I_{0}\left(G_{+}\right)$. Let $R_{G}=D_{0} \times Q\left(D_{+}\right)$or $D_{0} \times Q^{\prime}\left(D_{+}\right)$and $A_{G}(i):=A_{i}\left(D_{G}\right)=D_{0} \times A_{i}\left(D_{+}\right)$or $D_{0} \times A_{i}^{\prime}\left(D_{+}\right)$according as $D_{+} \nsucceq \mathbb{Z}_{2^{a+1+\alpha}}\left\langle\mathbb{Z}_{2}\right.$ for all $\alpha \geq 0$ or $D_{+}=\mathbb{Z}_{2^{a+1+\alpha}}\left\langle\mathbb{Z}_{2}\right.$ for some $\alpha \geq 0$, where $A_{i}\left(D_{+}\right)$and $A_{i}^{\prime}\left(D_{+}\right)$are given by (6.11) and (6.15), respectively. Then $A_{G}(i) \leq R_{G}, z_{c} \in A_{G}(1), R_{G}=\left\langle A_{G}(1), A_{G}(2)\right\rangle, C_{G}\left(A_{G}(i)\right)$ is regular, $s \in C_{G}\left(A_{G}(i)\right)$ and $T_{G}(i):=C_{C_{G}\left(A_{G}(i)\right)}(s)$ is a maximal torus given by (8.5).

Let $A_{c}(i)=A_{G}(i) \cap K_{c}, R_{c}=R_{G} \cap K_{c}$, and let $\left(A_{c}(i), b_{A_{c}(i)}\right) \leq\left(R_{c}, b_{c}\right)$ be $B_{c}$ subgroups, and $\left(A_{c}(i), b_{A_{G}(i)}\right) \leq\left(R_{c}, b_{G}\right) B_{G^{-}}$-subgroups such that $b_{A_{G}(i)}$ covers $b_{A_{c}(i)}$ and $b_{G}$ covers $b_{c}$, so that $z_{c} \in A_{c}(1)$ and $R_{c}=\left\langle A_{c}(1), A_{c}(2)\right\rangle$. Thus $T_{G}(i)=C_{C_{G}\left(A_{c}(i)\right)}(s)$, $C_{G}\left(R_{c}\right) \cap C_{G}(s)=C_{G}\left(R_{G}\right) \cap C_{G}(s), b_{A_{G}(i)}=\mathcal{E}_{2}\left(C_{G}\left(A_{c}(i)\right),(s)\right), D\left(b_{A_{G}(i)}\right)=O_{2}\left(T_{G}(i)\right)$, $D\left(b_{G}\right)=O_{2}\left(T_{G}(1) \cap C_{G}\left(R_{c}\right)\right), D\left(b_{A_{c}(i)}\right)=O_{2}\left(T_{c}(i)\right)$ and $D\left(b_{c}\right)=O_{2}\left(T_{c}(1) \cap C_{K_{c}}\left(R_{c}\right)\right)$, where $T_{c}(i)=T_{G}(i) \cap K_{c}$. Let $P_{c}=Z\left(R_{c}\right)$ and $\left(P_{c}, g_{c}\right) \leq\left(R_{c}, b_{c}\right)$. As shown in the proof (1) of Proposition 8.2, $\ell\left(g_{c}\right) \geq 2$ and there exists $y \in N_{C_{K_{c}}\left(P_{c}\right)}\left(R_{c}, b_{G}\right) \backslash C_{G}\left(R_{c}\right)$ such that $|y|=3$ and $y \in N_{C_{K_{c}}\left(P_{c}\right)}\left(R_{c}, b_{c}\right)$.

Let $T(i)$ be maximal torus of $K$ such that $T(i) / Z=T_{c}(i)$, and let $A(i)=O_{2}(T(i))$ and $R=\langle A(1), A(2)\rangle$. Then $A(i) / Z=A_{c}(i), R / Z=R_{c}$ and $R^{y}=R$. If $b_{A}(i)$ is the block of $C_{K}(A(i))$ dominating $b_{A_{c}(i)}$, then $b_{A}(i)=\mathcal{E}_{2}\left(C_{K}(A(i)),(s)\right)$ and so $\left(A(i), b_{A}(i)\right)$ is a $B$-subgroup as $B=\mathcal{E}_{2}\left(K,(s)\right.$ ) (note we identify $s$ with $s^{*}$ ). Similarly, if $b$ is the block of $C_{K}(R)$ dominating $b_{c}$, then $b=\mathcal{E}_{2}\left(C_{K}(R),(s)\right)$ and $(R, b)$ is a $B$-subgroup. But $b_{c}^{y}=b_{c}$, so by the uniqueness, $b^{y}=b$. Since $D(b) / Z=D\left(b_{c}\right)$ and $D\left(b_{c}\right) \leq T_{c}(1)$, it follows that $D(b) \leq T(1)$ and hence $D(b)$ is abelian. Let $P \leq Z(R)$ such that $P / Z=P_{c}$ and $g=\mathcal{E}_{2}\left(C_{K}(P),(s)\right)$. Then $g$ dominates $g_{c}$ and so $\ell(g)=\ell\left(g_{c}\right) \geq 2$.

It follows by Proposition 8.2 that $C_{H}(R) R / Z(K)=C_{H / Z(K)}(R / Z(K))$, and so $C_{H}(R) R / Z^{\prime}=C_{H / Z^{\prime}}\left(R / Z^{\prime}\right)$ for any $Z^{\prime} \leq Z(K)$. Similarly, $C_{H}(P) / Z^{\prime}=C_{H / Z^{\prime}}\left(P / Z^{\prime}\right)$.

Let $\left(R, b_{H}\right)$ be a $B_{H^{-}}$-subgroup such that $b_{H}$ covering $b$. Since $H / K Z(H)$ is a 2 group, it follows that $b_{H}$ is the unique block covering $b$, so that $b_{H}^{y}=b_{H}$. To show that 
$D\left(b_{H}\right)$ is abelian we may suppose $H / Z(H) K=J_{0}(V) / K_{c} Z\left(J_{0}(V)\right)=\operatorname{Outdiag}(K)$.

By $(8.5), T_{G}:=T_{G}(1)=\prod_{\Gamma}\left(\left(T_{\Gamma}^{\prime}\right)^{n_{\Gamma}} \times\left(T_{\Gamma}\right)^{m_{\Gamma}^{\prime}}\right)$, where $T_{\Gamma}^{\prime}=\operatorname{GL}^{\epsilon_{\Gamma}}\left(1, q^{\delta_{\Gamma}}\right), T_{\Gamma}=$ $\mathrm{GL}^{\epsilon_{\Gamma}}\left(1, q^{e_{\Gamma} \delta_{\Gamma}}\right)$ and $m_{\Gamma}^{\prime}=\left(m_{\Gamma}-n_{\Gamma}\right) / e_{\Gamma}$. Let $U_{\Gamma}$ and $W_{\Gamma}$ be orthogonal spaces over $\mathbb{F}_{q}$ such that $\operatorname{dim} U_{\Gamma}=d_{\Gamma}, \eta\left(U_{\Gamma}\right)=\epsilon_{\Gamma}$, and $\operatorname{dim} W_{\Gamma}=e_{\Gamma} d_{\Gamma}$ and $\eta\left(W_{\Gamma}\right)=\epsilon_{\Gamma}^{e_{\Gamma}}$. Let $J=J_{0}(V), B_{J}=\mathcal{E}_{2}(J,(s))$ and $T_{J}:=C_{J}\left(T_{G}\right)$. By [14, (1B)], $T_{J}=\left\langle T_{G}, \tau\right\rangle$ such that $\tau=\prod_{\Gamma}\left(\tau_{\Gamma}^{\prime} \times \tau_{\Gamma}\right)$ and $\left[\tau_{\Gamma}^{\prime}, T_{G}\right]=\left[\tau_{\Gamma}, T_{G}\right]=1$, where $\tau_{\Gamma}^{\prime} \in J_{0}\left(U_{\Gamma}\right)$ and $\tau_{\Gamma} \in J_{0}\left(W_{\Gamma}\right)$ such that $J_{0}\left(U_{\Gamma}\right)=\left\langle I_{0}\left(U_{\Gamma}\right), \tau_{\Gamma}^{\prime}\right\rangle$ and $J_{0}\left(W_{\Gamma}\right)=\left\langle I_{0}\left(W_{\Gamma}\right), \tau_{\Gamma}\right\rangle$. Thus for any $t_{c} \in \operatorname{Outdiag}(K)$ there exists an element $h \in T_{J}$ such that $h$ induces the same automorphism $t_{c}$ on $K$.

Since $H / Z(H)=J / Z(J)$, it follows that we may suppose $V=U_{\Gamma}$ or $W_{\Gamma}$, so that $C_{G}(s)=T_{\Gamma}^{\prime}=\mathrm{GL}^{\epsilon_{\Gamma}}\left(1, q^{\delta_{\Gamma}}\right)$ or $\mathrm{GL}^{\epsilon_{\Gamma}}\left(e_{\Gamma}, q^{\delta_{\Gamma}}\right)$. If $2 \|\left(q^{\delta_{\Gamma}}-\epsilon_{\Gamma}\right)$, then $e_{\Gamma}=2, C_{G}(s)=T_{\Gamma}^{\prime}$ or $\mathrm{GL}^{\epsilon_{\Gamma}}\left(2, q^{\delta_{\Gamma}}\right)$. If $4 \mid\left(q^{\delta_{\Gamma}}-\epsilon_{\Gamma}\right)$, then $e_{\Gamma}=1$ and $C_{G}(s)=T_{G}=\mathrm{GL}^{\epsilon_{\Gamma}}\left(1, q^{\delta_{\Gamma}}\right)$. Suppose there exists a primary element $z^{\prime} \in C_{G}(s)$ with $\left|z^{\prime}\right|=4$. Then $z^{\prime 2} \in Z(G)$, $C_{G}\left(z^{\prime}\right)=\mathrm{GL}^{\epsilon}(m, q)$ with $\operatorname{dim} V=2 m$ and so $C_{K}\left(z^{\prime}\right)$ and $C_{\operatorname{Inndiag}(K)}\left(z^{\prime}\right)$ are given by [16, Tables 4.5.1 and 4.5.2]. If $x \in C_{G}\left(z^{\prime}\right)$ with $|x|$ odd, then $x \in C_{K_{c}}\left(z^{\prime}\right)$. If $x=t_{x} Z$ for some $t_{x} \in K$ with odd order, then $t_{x}^{z^{\prime}}=t_{x}$ and $t_{x} \in C_{K}\left(z^{\prime}\right)$. Conversely, if $t_{x} \in C_{K}\left(z^{\prime}\right)$ with odd order, then $x:=t_{x} Z \in C_{K_{c}}\left(z^{\prime}\right)$. Since $\mathbb{F}_{q}$ has the odd characteristic $r$, it follows that $O^{r^{\prime}}\left(C_{G}\left(z^{\prime}\right)\right)=O^{r^{\prime}}\left(C_{K}\left(z^{\prime}\right)\right) Z / Z$ and in particular, $O^{r^{\prime}}\left(C_{K}\left(z^{\prime}\right)\right)=\operatorname{SL}^{\epsilon}(m, q)$. Since $T_{G}=T_{\Gamma}^{\prime}$ or $T_{\Gamma}$ which is a maximal torus of $C_{G}\left(z^{\prime}\right)$, it follows that $z^{\prime} \in T_{G}$ and $T_{G} \leq C_{G}\left(z^{\prime}\right)$. But $T_{J}=\left\langle T_{G}, \tau\right\rangle$, so by [14, (1B)], we may suppose $C_{J}\left(z^{\prime}\right)=C_{G}\left(z^{\prime}\right) T_{J}=$ $C_{G}\left(z^{\prime}\right) \circ\langle\tau\rangle$. Note that $N_{I(V)}\left(C_{G}\left(z^{\prime}\right)\right)=N_{I(V)}\left(\left\langle z^{\prime}\right\rangle\right)=\left\langle C_{G}\left(z^{\prime}\right), w\right\rangle$ and $N_{J}\left(\left\langle z^{\prime}\right\rangle\right) \leq$ $C_{J}\left(z^{\prime}\right) N_{I(V)}\left(\left\langle z^{\prime}\right\rangle\right)$, where $w \notin C_{G}\left(z^{\prime}\right)$ such that $w$ induces the graph automorphism on $O^{r^{\prime}}\left(C_{G}\left(z^{\prime}\right)\right)$, and $w \in G$ when $\eta(V)=+$ and $m$ is even. So the elements of $C_{J}\left(z^{\prime}\right)$ induce inner-diagonal automorphisms on $L:=O^{r^{\prime}}\left(C_{K}\left(z^{\prime}\right)\right)$. In addition, since $[\tau, x]=1$ for any $x \in C_{G}\left(z^{\prime}\right)$ with odd order, it follows that $\tau$ centralizes $L$.

Suppose $z^{\prime} \in K_{c}$, so that $z_{c}=z^{\prime}$, Let $z \in K$ such that $z Z=z_{c}$. Thus $C_{K}(z)=$ $C_{K}\left(z^{\prime}\right), C_{H}(z) / Z \leq C_{J}\left(z^{\prime}\right)$ and so elements of $C_{H}(z)$ induce inner-diagonal automorphisms on $L$. Now $C_{K}(z)$ is regular, $s \in C_{K}(z)$ and so $B_{z}=\mathcal{E}_{2}\left(C_{K}(z),(s)\right)$ is a block with a defect group $D\left(B_{z}\right)=O_{2}\left(C_{K}(z s)\right)=O_{2}(T(1))$. Let $B_{H_{z}}$ be a block of $C_{H}(z)$ such that $B_{H_{z}}$ covers $B_{z}$ and $b_{H}^{C_{H}(z)}=B_{H_{z}}$, so that $D\left(b_{H}\right) \leq D\left(B_{H_{z}}\right)$. Since $C_{H}(z)$ induces inner-diagonal automorphisms on $L$, it follows by Proposition 8.1 that $D\left(B_{H_{z}}\right)$ is abelian and so is $D\left(b_{H}\right)$.

Suppose $z^{\prime} \notin K_{c}$, so that $4\left\|\left(q^{\delta_{\Gamma}}-\epsilon_{\Gamma}\right), 4\right\|(q-\epsilon)$ and $C_{G}(s)=\mathrm{GL}^{\epsilon_{\Gamma}}\left(1, q^{\delta_{\Gamma}}\right)$ with $O_{2}\left(C_{G}(s)\right)=\mathbb{Z}_{4}$. Thus $D\left(b_{c}\right)=O_{2}\left(K_{c}\right) \leq Z(G)=\mathbb{Z}_{2}$. Note that $D\left(b_{c}\right)$ is also equal to $\mathrm{O}_{2}\left(K_{c}\right)$ when $C_{G}(s)$ has no primary element $z^{\prime}$ of order 4 .

If $D\left(b_{c}\right)=1$, then $D(b)=Z(K)=\mathbb{Z}_{2}, \operatorname{Outdiag}\left(K_{c}\right)=\mathbb{Z}_{2},\left|D\left(b_{H}\right)\right| \leq 4$ and $D\left(b_{H}\right)$ is abelian. Suppose $D\left(b_{c}\right)=O_{2}\left(K_{c}\right)=\mathbb{Z}_{2}$. Then $D(b)=O_{2}(K)=\mathbb{Z}_{2} \times \mathbb{Z}_{2}$ or $\mathbb{Z}_{4}$ according as $G=\mathrm{SO}^{+}(4 k, q)$ or $G=\mathrm{SO}^{\epsilon}(4 k+2, q)$. In the former case, $C_{G}(s)=$ $\operatorname{GL}\left(1, q^{2 k}\right)$ and $O_{2}\left(C_{G}(s)\right)=\left\langle z^{\prime \prime}\right\rangle$ with $\left|z^{\prime \prime}\right|=2^{\ell} \geq 8$, and hence $z^{\prime}=z^{\prime 2^{\ell-2}} \in K_{c}$, which is impossible.

Thus $G=\operatorname{SO}^{\epsilon}(2 m, q)$ with $m=2 k+1$ and $4 \|(q-\epsilon)$. Suppose $t \in D\left(b_{H}\right) \backslash D(b)$ induces an element of order 4 in $\operatorname{Outgiag}(K)$. Then there exists $x_{t} \in J$ such that $x_{t}$ induces the same action as $t$ on $K_{c} / Z$. Since $J / G \cong \mathbb{Z}_{q-\epsilon}$, it follows by [16, Table 4.5.2] that we may suppose $x_{t} \in J \backslash G$ such that $x_{t}^{4} \in Z(G), x_{t}^{2} \in G Z(J)$ and $C_{K}\left(x_{t}^{2}\right)=$ $C_{K}\left(t^{2}\right)=\left(\mathrm{SL}^{\epsilon}(m, q) \circ \mathbb{Z}_{q-\epsilon}\right) \cdot(\operatorname{gcd}(m, q-\epsilon))$. Thus $C_{G}\left(x_{t}\right)=C_{G}\left(x_{t}^{2}\right)=\operatorname{GL}^{\epsilon}(m, q)$ and $x_{t}$ centralizes elements of odd order in $C_{K}\left(x_{t}^{2}\right)$. In particular, $\left[x_{t}, L\right]=[t, L]=1$ with $L=O^{r^{\prime}}\left(C_{G}\left(t^{2}\right)\right)=\mathrm{SL}^{\epsilon}(m, q)$. By [16, Table 4.5.1], elements of $C_{\operatorname{Inndiag}(K)}\left(t^{2}\right)$ induce 
inner-diagonal automorphism on $L$. Let $B_{t}=\mathcal{E}_{2}\left(C_{K}\left(t^{2}\right),(s)\right)$ and $B_{H_{t}} \in \operatorname{Blk}\left(C_{H}\left(t^{2}\right)\right)$ such that $B_{H_{t}}$ covers $B_{t}$ and $b_{H}^{C_{H}\left(t^{2}\right)}=B_{H_{t}}$, so that $D\left(B_{t}\right)=O_{2}\left(C_{C_{K}\left(t^{2}\right)}(s)\right)=Z(K)$. By Proposition 8.1, $D\left(B_{H_{t}}\right)$ is abelian and so is $D\left(b_{H}\right)$. If $D\left(b_{H}\right) / D(b)=\mathbb{Z}_{2}$, then as shown above $D\left(b_{H}\right)=\left\langle Z(K), t^{2}\right\rangle$ is abelian.

(2). Suppose $D$ is abelian, so that $D_{G}$ is abelian, $C_{K}(s)$ is a maximal torus of $K$ and by $[14,(2 \mathrm{E})], C_{D_{0}(V)}(s)$ is a maximal torus of $D_{0}(V)$. To show that $D\left(B_{H}\right)$ is abelian we may suppose $H / K Z(H)=J_{0}(V) / K_{c} Z\left(J_{0}(V)\right)=\operatorname{Outdiag}(K)$. So the proof is given in (1) above, and Property 7.1 (b) holds.

Theorem 8.7 Let $K$ be a finite quasi-simple group of classical type over a field $\mathbb{F}_{q}$ and $B \in \operatorname{Blk}(K)$, and let $K \triangleleft H$ such that $H / K$ is abelian, $C_{H}(K) \leq Z(H), H$ induces inner-diagonal automorphisms on $K$ and $B_{H} \in \operatorname{Blk}(H)$ covering $B$. If $q$ is even, then either $D(B)=D\left(B_{H}\right)$ is cyclic or $\ell(B) \geq 2$. Suppose $q$ is odd. Then either Property 7.1 (a) or (b) holds.

Proof: We will follow the notation of [16]. In particular, $K_{u}$ denotes the universal group with the same type as $K$ and $K=K_{u} / Z$ for some $Z \leq Z\left(K_{u}\right)$. If $q$ is even and $D(B)$ is noncyclic, then $D(B)$ is a Sylow subgroup of $K$ and $\ell(B)=\ell\left(B_{0}\right)$ with principal $B_{0}:=B_{0}(K) \in \operatorname{Blk}(K)$. But $B_{0}$ dominates the principal block $\bar{B}$ of $K / Z(K)=K_{a}$ and $\ell(\bar{B})+1$ is the number of $2^{\prime}$-conjugacy classes of $K_{a}$, so $\ell\left(B_{0}\right) \geq \ell(\bar{B}) \geq 2$.

Suppose $q$ is odd. If $K=A_{n}^{\eta}(q)$, then take $\widehat{K}=K_{u}=\operatorname{SL}_{n+1}^{\eta}(q) \leq \widehat{H} \leq \operatorname{GL}_{n+1}^{\eta}(q)$ such that $H=\widehat{H} / Z$.

If $K=B_{n}(q)=K_{a}=\Omega_{2 n+1}(q)$, then set $\widehat{K}=\Omega_{2 n+1}(q) \leq \widehat{H} \leq \operatorname{SO}_{2 n+1}(q)$ such that $H=\widehat{H} / Z$. If $K=B_{n}(q)=K_{u}=\operatorname{Spin}_{2 n+1}(q)=\operatorname{Spin}(V)$, then take $K=\widehat{K} \triangleleft \widehat{H}=H$ such that $H / Z(K) \leq \mathrm{SO}(V)$.

If $K=C_{n}(q)$, then we may take $\widehat{K}=\operatorname{Sp}_{2 n}(q)=\operatorname{Sp}(V) \leq \widehat{H} \leq J_{0}(V)$ such that $H=\widehat{H} / Z$.

Suppose $K=D_{n}^{\eta}(q)$ with $(n, \eta)=(2 k+1, \pm)$ or $(2 k,-)$. If $K=\Omega_{2 n}^{\eta}(q)=\Omega(V)$, then $K=\widehat{K} \triangleleft \widehat{H}=H \leq J_{0}(V)$. If $K=P \Omega_{2 n}^{\eta}(q)=P \Omega(V)$, then take $\widehat{K}=$ $\Omega_{2 n}^{\epsilon}(q) \leq \widehat{H} \leq J_{0}(V)$ such that $H=\widehat{H} / Z$. If $K=\operatorname{Spin}_{2 n}^{\eta}(q)=\operatorname{Spin}(V)$, then take $K=\widehat{K} \triangleleft \widehat{H}=H$ such that $H / Z(K) \leq J_{0}(V)$.

Suppose $K=D_{2 k}^{+}(q)$, so that $Z\left(K_{u}\right)=\left\{1, z, z_{s}, z_{c}\right\}$ and $K_{u} /\langle z\rangle=\Omega_{4 k}^{+}(q)$. If $K=\Omega_{4 k}^{+}(q)=\Omega(V)$, then take $\widehat{K}=K \leq \widehat{H} \leq J_{0}(V)$. If $K=P \Omega_{4 k}^{+}(q)=P \Omega(V)$, then take $\widehat{K}=\Omega(V) \leq \widehat{H} \leq J_{0}(V)$ such that $\widehat{H} / Z=H$ with $Z=\langle z\rangle$. If $K=\operatorname{Spin}_{4 k}^{+}(q) / Z^{\prime}$ for $Z^{\prime}=\left\langle z_{s}\right\rangle$ or $\left\langle z_{c}\right\rangle$, then we may take $\widehat{K}=\operatorname{Spin}_{4 k}^{+}(q)=\operatorname{Spin}(V) \leq \widehat{H} \leq D_{0}(V)$ such that $H=\widehat{H} / Z^{\prime}$. If $K=\operatorname{Spin}_{4 k}^{+}(q)=\operatorname{Spin}(V)$, then take $\widehat{K}=K$ and $\widehat{H}=H$.

Let $\widehat{B} \in \operatorname{Blk}(\widehat{K})$ dominating $B$ and $\widehat{B}_{H} \in \operatorname{Blk}(\widehat{H})$ dominating $B_{H}$, so that $\widehat{B}_{H}$ covers $\widehat{B}$. By Propositions 8.1, 8.2, 8.4 and 8.6, one of Properties 7.1 (a) and (b) holds for $\widehat{B}$.

If Property 7.1 (a) holds for $\widehat{B}$, then there exist $\widehat{B}$-subgroups $(\widehat{P}, \hat{g}) \leq(\widehat{R}, \hat{b})$ satisfying Property 7.1 (a), where $\widehat{P} \leq Z(\widehat{R})$. By Lemma 7.3, Property 7.1 (a) holds for some $B$-subgroups $(P, g) \leq(R, b)$.

If Property 7.1 (b) holds for $\widehat{B}$, then $D(\widehat{B})$ and $D\left(\widehat{B}_{H}\right)$ are both abelian. Since $Z \leq Z(\widehat{H}) \cap \widehat{K}$, it follows that $D(B)=D(\widehat{B}) Z / Z$ and $D\left(B_{H}\right)=D\left(\widehat{B}_{H}\right) Z / Z$, and so 
$D(B)$ and $D\left(B_{H}\right)$ are both abelian.

\section{$9 \quad$ Exceptional groups}

We will follow the notation of [16].

Theorem 9.1 Let $K$ be a finite quasi-simple group of exceptional type over a field $\mathbb{F}_{q}, B \in \operatorname{Blk}(K)$, and let $K \triangleleft H$ such that $C_{H}(K) \leq Z(H), H / K$ is cyclic, and $H$ induces inner-diagonal automorphisms on $K$. If $q$ is even, then either $D(B)=D\left(B_{H}\right)$ is cyclic or $\ell(B) \geq 2$. If $q$ is odd, then either Property 7.1 (a) or (b) holds.

Proof: If $q$ is even, then a proof similar to that of Theorem 8.7 shows that either $D(B)=D\left(B_{H}\right)$ is cyclic or $\ell(B) \geq 2$.

Suppose $q$ is odd. Let $K_{u}$ be the universal group, so that $K=K_{u} / Z$ for some $Z \leq Z\left(K_{u}\right)$. Since $Z\left(K_{u}\right)$ is cyclic of order 1,2 or 3 , it follows that $H$ centralizes $Z\left(K_{u}\right)$.

Let $D:=D(B)$. If $Z(K) \neq \Omega_{1}(Z(D))$, then take an involution $z \in Z(D) \backslash Z(K)$. If $Z(K)=\Omega_{1}(Z(D))$, then take $z \in D$ such that $|z|=4$ and $z Z(K) \in Z(D / Z(K))$. Let $\left(z, B_{z}\right)$ be a $B$-subsection, and in the case $z \in Z(D)$ we take $B_{z}$ to have defect group $D$ by $[1,4.15]$. Write $C:=C_{G}(z)$.

By [16, Theorem 4.2.2] $C=O^{r^{\prime}}(C) T$, where $O^{r^{\prime}}(C)$ is a central product

$$
O^{r^{\prime}}(C)=L_{1} \circ L_{2} \circ \cdots \circ L_{\ell}
$$

with each $L_{i} \in \mathcal{L} i e(r)$, and $T$ is an abelian $r^{\prime}$-group inducing inner-diagonal automorphisms on each $L_{i}$. In general, it may be the case that $z \notin O^{r^{\prime}}(C)$. We introduce some more notation as follows to allow for this inconvenience: If $Z(C) \leq O^{r^{\prime}}(C)$, then define $s:=\ell$ and $L:=O^{r^{\prime}}(C)$. If $Z(C) \not \leq O^{r^{\prime}}(C)$, then define $s=\ell+1, L_{s}=Z(C)$ and

$$
L:=L_{1} \circ L_{2} \circ \cdots \circ L_{s} .
$$

In all cases $C=L T, z \in L$ and $L \triangleleft C$. Let $B_{L}$ be a block of $L$ covered by $B_{z}$. There are uniquely defined blocks $B_{i} \in \operatorname{Blk}\left(L_{i}\right)$ such that if $\chi \in \operatorname{Irr}\left(B_{L}\right)$ with $\chi=\chi_{1} \circ \cdots \circ \chi_{s}$ for some $\chi_{i} \in \operatorname{Irr}\left(L_{i}\right)$, then $\chi_{i} \in \operatorname{Irr}\left(B_{i}\right)$. We write

$$
B_{L}=B_{1} \circ B_{2} \circ \cdots \circ B_{s}
$$

Case 1. Suppose that $H=K:={ }^{2} G_{2}\left(3^{2 a+1}\right), G_{2}(q),{ }^{3} D_{4}(q), F_{4}(q)$ or $E_{6}^{-\epsilon}(q)$ with $q \equiv \epsilon \quad(\bmod 3)$, and $B \in \operatorname{Blk}(K)$. Then either Property $7.1(a)$ or $(b)$ holds.

Since $z$ induces an inner automorphism on $K$, it follows that each $L_{i}$ is a classical group (with possibly $L_{s}$ abelian).

If $\ell=1$, then $L_{1}$ is a classical group given by [16, Table 4.5.1]. Thus Theorem 9.1 follows by Theorem 8.7.

Suppose $\ell \geq 2$, so that by [16, Table 4.5.1], $\ell=2, Z(C) \leq L_{1} \circ L_{2}, s=\ell$, $L=L_{1} \circ L_{2}$ and the possible $(K, C)$ are given in Table 2 , where $\bar{\eta}=-$ or + . Here 


\begin{tabular}{|l|l|l|l|}
\hline $\mathrm{K}$ & $C$ & $K$ & $C$ \\
\hline${ }^{3} D_{4}(q)$ & $\left(\mathrm{SL}_{2}(q) \circ \mathrm{SL}_{2}\left(q^{3}\right)\right) \cdot(2: 2)$ & $G_{2}(q)$ & $\left(\mathrm{SL}_{2}(q) \circ \mathrm{SL}_{2}(q)\right) \cdot(2: 2)$ \\
$F_{4}(q)$ & $\left(\mathrm{SL}_{2}(q) \circ \mathrm{Sp}_{6}(q)\right) \cdot(2: 2)$ & $E_{6}^{\eta}(q)_{u}$ & $\left(\mathrm{SL}_{2}(q) \circ \mathrm{SL}_{6}^{\eta}(q)\right) \cdot(2: 2)$ \\
$\left.E_{7}(q)\right)_{u}$ & $\left(\mathrm{SL}_{2}(q) \circ \mathrm{Spin}_{12}^{+}(q)\right) \cdot(2: 2)$ & $E_{8}(q)$ & $\left(\mathrm{SL}_{2}(q) \circ E_{7}(q) u\right) \cdot(2: 2)$ \\
\hline
\end{tabular}

Table 2: Possible $(K, C)$ with $s \geq 2$

$C=\left(L_{1} \circ L_{2}\right) \cdot(2: 2)$ means that $C=\left\langle L_{1} \circ L_{2}, x\right\rangle$ such that $x$ induces inner-diagonal automorphism of order 2 on each $L_{i}$.

Write $L_{1}:=\mathrm{SL}_{2}(q) \leq H_{1}:=\left\langle L_{1}, x_{1}\right\rangle \leq G_{1}=\mathrm{GL}_{2}^{\delta}(q), L_{2}=\mathrm{SL}_{2}\left(q^{3}\right), \mathrm{SL}_{2}(q)$, $\mathrm{Sp}_{6}(q)$ or $\mathrm{SL}_{6}^{-\epsilon}(q)$ and $H_{2}=\left\langle L_{2}, x_{2}\right\rangle$, where $\delta$ is the sign such that $2 \|(q-\delta 1)$ and $x=x_{1} \times x_{1} \in C \backslash L$ such that $x_{i}$ induces outer-diagonal automorphism of order 2 on $L_{i}$. Then $C \triangleleft H:=H_{1} \circ H_{2}$. If $B_{H} \in \operatorname{Blk}(H)$ covers $B_{z}$, then $B_{H}$ covers $B_{L}$, $B_{H}=B_{H_{1}} \circ B_{H_{2}}$ for some $B_{H_{i}} \in \operatorname{Blk}\left(H_{i}\right)$ covering $B_{i}, D\left(B_{H}\right)=D\left(B_{H_{1}}\right) \circ D\left(B_{H_{1}}\right)$ and $D\left(B_{z}\right)=D\left(B_{H}\right) \cap C$. Each $B_{i}$ satisfies Property 7.1 (a) or (b).

If both $B_{1}$ and $B_{2}$ satisfy (b), then $B_{z}$ satisfies (b).

Suppose both $B_{1}$ and $B_{2}$ satisfy Property 7.1 (a), for $\left(P_{1}^{\prime}, g_{1}^{\prime}\right) \leq\left(R_{1}^{\prime}, b_{1}^{\prime}\right)$ and $\left(P_{2}, g_{2}\right) \leq\left(R_{2}, b_{2}\right)$, respectively. Then $R_{1}^{\prime}$ is non-abelian in $L_{1}=\mathrm{SL}_{2}(q)$ and so $b_{1}^{\prime}$ is principal. Thus $P_{1}^{\prime}=Z\left(L_{1}\right)$ and $g_{1}^{\prime}$ is principal. Let $Q_{1} \in \operatorname{Syl}_{2}\left(H_{1}\right)$ containing $R_{1}^{\prime}$, so that $Q_{1}=S D_{2^{a+2}}$. Writing $S_{1}=C_{Q_{1}}\left(\left[Q_{1}, Q_{1}\right]\right)$ and $P_{1}=S_{1} \cap L_{1}$, we have $S_{1} \cong \mathbb{Z}_{2^{a+1}}$, $P_{1} \cong \mathbb{Z}_{2^{a}}, C_{G_{1}}\left(P_{1}\right) \cong \mathbb{Z}_{q^{2}-1}$ and $C_{L_{1}}\left(P_{1}\right) \cong \mathbb{Z}_{q+\delta}$. Set $P=P_{1} \circ P_{2}, R=P_{1} \circ R_{2}$, $g_{1}=b_{1}=B_{0}\left(C_{L_{1}}\left(P_{1}\right)\right)$ and $y=y_{1} \times y_{2}$, where $y_{1}=1$ and $y_{2} \in N_{C_{L_{2}}\left(P_{2}\right)}\left(R_{2}, b_{2}\right) \backslash C_{L_{2}}\left(R_{2}\right)$ with $\left|y_{2}\right|=3$.

Suppose one of the $B_{i}$ satisfies Property 7.1 (a) and the other (b). Say $B_{1}$ satisfies Property 7.1 (a) for $\left(P_{1}, g_{1}\right) \leq\left(R_{1}, b_{1}\right)$, and $B_{2}$ satisfies Property 7.1 (b). Then let $\left(P_{2}, g_{2}\right)=\left(R_{2}, b_{2}\right)$ be a Sylow $B_{2}$-subgroup, and define $P$ and $R$ as above, and $y=$ $y_{1} \times y_{2}$ with $y_{1} \in N_{C_{L_{1}}\left(P_{1}\right)}\left(R_{1}, b_{1}\right) \backslash C_{L_{1}}\left(R_{1}\right)$ such that $\left|y_{1}\right|=3$ and $y_{2}=1$.

In either case $\left(P, g_{1} \circ g_{2}\right) \leq\left(R, b_{1} \circ b_{2}\right)$ are $B_{L^{-}}$subgroups. Let $(P, g) \leq(R, b)$ be $B_{z^{-}}$ subgroups such that $g$ and $b$ cover $g_{1} \circ g_{2}$ and $b_{1} \circ b_{2}$, respectively. Let $\left(P, g_{H}\right) \leq\left(R, b_{H}\right)$ be $B_{H^{-}}$-subgroups such that $g_{H}$ and $b_{H}$ cover $g$ and $b$, respectively. Then $g_{H}=g_{H_{1}} \circ g_{H_{2}}$ for some $g_{H_{i}} \in \operatorname{Blk}\left(C_{H_{i}}\left(P_{i}\right)\right)$ covering $g_{i}$, and $b_{H}=b_{H_{1}} \circ b_{H_{2}}$ for some $b_{H_{i}} \in \operatorname{Blk}\left(C_{H_{i}}\left(R_{i}\right)\right)$ covering $b_{i}$. By Propositions 8.1 and 8.4, each $D\left(b_{H_{i}}\right)$ is abelian and $b_{H_{i}}^{y_{i}}=b_{H_{i}}$. So $D(b)=\left(D\left(b_{H_{1}}\right) \circ D\left(b_{H_{2}}\right)\right) \cap C_{C}(R)$ is abelian and $b_{H}^{y}=b_{H}$. Since $b_{H}$ covers $b$ and $\left[C_{H}(R): C_{C}(R)\right] \leq 2$ and since $|y|=3$, it follows that $b^{y}=b$.

By consideration of the Lie rank of $L_{i}$, we have that $D\left(g_{i}\right) \neq \mathbb{Z}_{2^{a+\alpha}} \backslash \mathbb{Z}_{2}$ for any $\alpha \geq 1$. It follows that $D(g) / O_{2}\left(C_{C}(P)\right)$ is $\mathbb{Z}_{2} \times \mathbb{Z}_{2}$ or a dihedral group. By Lemma 2.4, $\ell(g) \geq 2$ and Property 7.1 (a) holds for $(P, g) \leq(R, b)$.

Case 2. Suppose $3 \mid(q-\epsilon)$, and let $K=K_{u}:=3 . E_{6}^{\epsilon}(q) \leq J:=3 . E_{6}^{\epsilon}(q) .3$, $B \in \operatorname{Blk}(K)$ and $B_{J} \in \operatorname{Blk}(J)$ covering $B$. Then either Property $7.1(a)$ or $(b)$ holds.

Write $m^{*}:=\operatorname{gcd}(m, q-\epsilon)$ and let $z \in Z(D)$ with $|z|=2$. By [16, Table 4.5.2], $C:=C_{K}(z)=\left\langle\operatorname{Spin}_{10}^{\epsilon}(q) \circ(q-\epsilon), t\right\rangle$ or is given in Table 2, where $t=4^{*}: 1$. By [16, Table 4.5.1], $C_{J}(z)=\left\langle\operatorname{Spin}_{10}^{\epsilon}(q) \circ(q-\epsilon), t_{J}\right\rangle$ or $\left\langle\mathrm{SL}_{2}(q) \circ \mathrm{SL}_{6}^{\epsilon}(q), x_{J}\right\rangle$, where $t_{J}=4^{*}: 3$ and $x_{J}=2: 6$. As in Case $1 \ell \leq 2$, and if $\ell=1$, then we are done by Theorem 8.7. If $\ell=2$, 
then define $L_{i}, B_{i}, H_{i}$ and $B_{H_{i}}$ analogously to Case 1. Property 7.1 (a) or (b) holds for each $B_{i}$, and if (a) holds for one or both of $B_{1}$ and $B_{2}$, then a proof similar to that of Case 1 shows that either Property 7.1 (a) holds for some $B$-subgroups $(P, g) \leq(R, b)$ or Property $7.1(b)$ holds for $B$.

Suppose Property 7.1 (b) holds for $B_{1}$ and $B_{2}$, and suppose $D_{J} \cap K=D$ for some $D_{J}=D\left(B_{J}\right)$, so that $D\left(B_{H_{i}}\right)$ is abelian and so is $D\left(B_{z}\right)=D\left(B_{H}\right) \cap C$. Now $B_{z}$ is major and so $D=D\left(B_{z}\right)=D_{J}$ is abelian. Thus Property 7.1 (b) holds for $B$.

Case 3. Let $K=2 . E_{7}(q) \leq J:=2 \cdot E_{7}(q) \cdot 2, B \in \operatorname{Blk}(K)$ and $B_{J} \in \operatorname{Blk}(J)$ covering $B$, where $q$ is odd. Then either Property $7.1(a)$ holds for some $B$-subgroups $(P, g) \leq(R, b)$ with $C_{J}(P) / Z=C_{J / Z}(P / Z)$ and $C_{J}(R) R / Z=C_{J / Z}(R / Z)$, or Property 7.1 (b) holds for $B$, where $Z=Z(K)$.

Again write $m^{*}:=\operatorname{gcd}(m, q-\epsilon)$. Since $z$ induces an inner automorphism on $K$, it follows by [16, Table 4.5.2] that

$$
C_{K}(z)=\left\langle\mathrm{SL}_{2}(q) \circ \operatorname{Spin}_{12}(q), t\right\rangle \quad \text { with } \quad t=2: 2,
$$

$\left\langle\left(\mathrm{SL}_{8}^{\epsilon}(q) / 2\right) \circ 4, x\right\rangle$ with $x=\left(8^{*} / 4\right): 1$ or $\left\langle E_{6}^{\epsilon}(q)_{u} \circ(q-\epsilon), w\right\rangle$ with $w=3^{*}: 1$, where the $\operatorname{sign} \epsilon= \pm$ is chosen so that $4 \mid(q-\epsilon)$.

Using Propositions 8.1 and 8.6 or Case 2, and applying a proof similar to that of Case 1, we have that if Property 7.1 (a) holds for some $B_{i}$-subgroups $\left(P_{i}, g_{i}\right) \leq\left(R_{i}, b_{i}\right)$, then the first part of Property 7.1 ( $a$ ) holds for some $B_{z}$-subgroup $(P, g) \leq(R, b)$.

Let $\left(R, b_{J}\right)$ be a $B_{J}$-subgroup such that $b_{J}$ covers $b$. By [16, Tables 4.5.1 and 4.5.2],

$$
C_{J}(z)=\left\langle\mathrm{SL}_{2}(q) \circ \operatorname{Spin}_{12}(q), t, t_{J}\right\rangle, \quad \text { with } \quad t=2: 2, \quad t_{J}=1: 2,
$$

$\left\langle\left(\mathrm{SL}_{8}^{\epsilon}(q) / 2\right) \circ 4, x_{J}\right\rangle$ with $x_{J}=\left(8^{*} / 2\right): 1$ or $\left\langle E_{6}^{\epsilon}(q)_{u} \circ(q-\epsilon) .2, w\right\rangle$ with $w$ given above. Thus $R \leq C_{J}(z), C_{J}(z)=L A$ for some abelian $A$ and $A$ induces inner-diagonal automorphism on each $L_{i}$. A proof similar to that of Case 1 shows that $D\left(b_{J}\right)$ is abelian. If $y \in N_{C_{K}(P)}(R, b) \backslash C_{K}(R)$ such that $|y|=3$, then $b_{J}^{y}=b_{J}$ since $\left|C_{J}(R): C_{K}(R)\right| \leq 2$ and $b_{J}$ is the unique cover of $b$.

Suppose $z \in D$ is such that $|z|=4, z^{2} \in Z$ and $z Z \in Z(D / Z)$. Then $4 \mid(q-\epsilon)$ and

$$
C_{J / Z}(z Z)=\left\langle\mathrm{SL}_{8}^{\epsilon}(q) / 4 \circ 2, \bar{x}_{J}, \bar{x}\right\rangle \quad \text { with } \quad \bar{x}_{J}=\left(8^{*} / 2\right): 1, \quad \bar{x}=\gamma
$$

or $\left\langle 3^{*} . E_{6}^{\epsilon}(q) \circ(q-\epsilon), \bar{w}, \bar{v}\right\rangle$ with $\bar{w}=3^{*}: 1$ and $\bar{v}=\gamma: i$. Here $\gamma$ and $i$ are graph and inverse automorphisms, respectively. Since $D \leq K$ and $D / Z \leq C_{J / Z}(z Z)$, it follows that $D / Z \leq C_{J}(z) / Z, z \in Z(D)$ and so $\left(z, B_{z}\right)$ is major.

If $L=\mathrm{SL}_{8}^{\epsilon}(q) / 2$ or $E_{6}^{\epsilon}(q)_{u}$, then by Proposition 8.1 or Case $2, C_{J}(P) / Z=C_{J / Z}(P / Z)$ and $C_{J}(R) R / Z=C_{J / Z}(R / Z)$. Suppose $L=\operatorname{SL}_{2}(q) \circ \operatorname{Spin}_{12}(q)$. By [16, Table 4.5.1],

$$
C_{J / Z}(z Z)=\left\langle\mathrm{SL}_{2}(q) \circ\left(\operatorname{Spin}_{12}(q) / 2\right), t, t_{J}\right\rangle
$$

where $t$ and $t_{J}$ are given above. Thus $C_{J}(z) / Z=C_{J / Z}(z Z)$ and by Propositions 8.1 and 8.6, $C_{J}(P) / Z=C_{J / Z}(P / Z)$ and $C_{J}(R) R / Z=C_{J / Z}(R / Z)$.

Suppose $D\left(B_{i}\right)$ and $D\left(B_{H_{i}}\right)$ are both abelian. Then $D=D\left(B_{z}\right)$ is abelian. Let $B_{J}$ cover $B$ and $D_{J}=D\left(B_{J}\right)$. If there exists $k \in Z\left(D_{J}\right) \backslash D$, then $k \in C_{J}(D) \backslash K$ 
and so $D_{J}=\langle D, k\rangle$ is abelian. If $Z\left(D_{J}\right) \leq D$, then we may choose $z \in Z\left(D_{J}\right)$ and so $D_{J} \leq C_{J}(z)=L A$. A proof similar to that of Case 1 with $R$ replaced by $D_{J}$ shows that $D_{J}$ is abelian and Property $7.1(b)$ holds.

Case 4. Suppose $K:=E_{8}(q)$, so that $\left(z, B_{z}\right)$ is a major subsection of $B$. Either $L_{i}$ is classical, or $L_{i}$ is exceptional and given in Cases 1, 2 or 3. If $L_{i}$ is classical, then apply Theorem 8.7. If $L_{i}$ is exceptional, then apply the results in Cases 1,2 or 3. A proof similar to that of Case 1 shows that either Property 7.1 (a) holds for $B$ or each $D\left(B_{i}\right)$ is abelian. In the latter case, each $D\left(B_{H_{i}}\right)$ is abelian, and so $D=D\left(B_{z}\right)=D\left(B_{H}\right) \cap C$ is abelian.

Lemma 9.2 Let $G$ be a quasisimple group such that $G / Z(G)$ is alternating or of Lie type, and $G$ is an exceptional cover. Then every 2-block of $G$ with nonabelian defect groups has at least two irreducible Brauer characters.

Proof: It suffices to consider the cases where $|Z(G)|$ is odd. We must consider the cases $G / Z(G) \cong A_{7}, P S L_{2}(9), P S U_{4}(3)$ and $O_{7}(3)$. In each case we may use [15] to confirm the result.

\section{Proofs of the main theorems}

We may finally prove that nilpotent 2-blocks of quasisimple groups have abelian defect groups:

Proof of Theorem 1.1. If $G / Z(G)$ is an alternating group, then the result follows by Corollary 3.3. For $G / Z(G)$ sporadic see Corollary 4.2. If $G / Z(G)$ is a classical group and $G$ is a non-exceptional cover, see Propositions 8.1, 8.2, 8.4 and 8.6. For $G / Z(G)$ an exceptional group of Lie type and $G$ is a non-exceptional cover, see Theorem 9.1. For the exceptional covers, see Lemma 9.2.

We complete the proof of Puig's conjecture for quasisimple groups for the prime 2:

Proof of Theorem 1.3. The necessary condition for nilpotency follows from [10, 1.2]. By Propositions 7.2, 8.1, 8.2, 8.4 and 8.6 the result holds for the classical groups. By Theorem 9.1 it holds for the exceptional groups of Lie type. The result holds for the double covers of the alternating groups by Proposition 3.4, and when $G / Z(G)$ is sporadic by Corollary 4.3. For the exceptional covers of the alternating groups and of the finite simple groups of Lie type, see Lemma 9.2.

Acknowledgement. We thanks the referee for their useful observations and suggestions. The second author also thanks the Department of Mathematics at the University of Auckland for its hospitality during visits in which part of this research was completed. 


\section{References}

[1] J. L. Alperin and M. Broué, Local methods in block theory, Ann. Math. 110 (1979), 143-157.

[2] J. An, 2-weights for general linear groups and 2-blocks of classical groups, PhD thesis of the University of Illinois at Chicago, 1991.

[3] J. An, 2-weights for classical groups, J. reine angew. Math. 439 (1993), 159-204.

[4] J. An, Weights for classical groups, Tran. Amer. Math. Soc. 342(1994), 1-42.

[5] J. An, J. J. Cannon, E. A. O'Brien and W. R. Unger, The Alperin weight conjecture and Dade's conjecture for the simple group $F i_{24}^{\prime}$, LMS J. Comput. Math. 11(2008), 100-145.

[6] J. An and C. W. Eaton, Nilpotent blocks of quasisimple groups for odd primes, $J$. reine angew. Math., to appear.

[7] J. An and E. A. O'Brien, The Alperin and Dade conjectures for the Conway simple group Co $\mathrm{Co}_{1}$ Alg. and Repr. Theory 7(2004), 139-158.

[8] J. An, E. A. O'Brien and R. A. Wilson, The Alperin weight conjecture and Dade's conjecture for the simple group $J_{4}, L M S$ J. Comput. Math. 6(2003), 119-140.

[9] M. Broué, Les $\ell$-blocs des groupes $\operatorname{GL}(n, q)$ et $\mathrm{U}\left(n, q^{2}\right)$ et leurs structures locales, Séminaire Bourbaki Astérisque 640 (1986), 159-188.

[10] M. Broué and L. Puig, A Frobenius theorem for blocks, Invent. Math. 56 (1980), 117-128.

[11] M. Cabanes and M. Enguehard, Representation Theory of Finite Reductive Groups (Cambridge University Press, 2004).

[12] J. H. Conway, R. T. Curtis, S. P. Norton, R. A. Parker and R. A. Wilson, Atlas of Finite Groups (Clarendon Press, Oxford, 1985).

[13] P. Fong and B. Srinivasan, The blocks of finite general linear and unitary groups, Invent. Math 69 (1982), 109-153.

[14] P. Fong and B. Srinivasan, The blocks of finite classical groups, J. reine angew. Math. 396 (1989), 122-191.

[15] The GAP Group, GAP - Groups, algorithms, and programming, version 4.4, http://www.gap-system.org, 2005.

[16] D. Gorenstein, R. Lyons and R. Solomon, The Classification of Finite Simple Groups. Number 3 (Mathematical Surveys and Monographs, AMS, Providence, 1998).

[17] A. Granville and K. Ono, Defect zero p-blocks for finite simple groups, Trans. AMS 348 (1996), 331-347. 
[18] G. James and A. Kerber, The representation theory of the symmetric group. (Addison-Wesley, 1981).

[19] C. Jansen, K. Lux, R. Parker and R. Wilson, An Atlas of Brauer Characters (Oxford Science Publication, Clarendon Press Oxford, 1995).

[20] O. Krull and P. Landrock, The characters of some 2-blocks of the babymonster, its covering group and the monster, Comm. Algebra 6 (1978), 1893-1921.

[21] B. Külshammer, On 2-blocks with wreathed defect groups, J. Algebra 64 (1980), 529-555.

[22] B. Külshammer and L. Puig, Extensions of nilpotent blocks, Invent. Math. 102 (1990), 17-71.

[23] P. Landrock, The non-principal 2-blocks of sporadic simple groups, Comm. Algebra 8(1978),1865-1891.

[24] G. Malle and G. Navarro, Blocks with equal height zero degrees, preprint (2009).

[25] H. Nagao and Y. Tsushima, Representations of finite groups, (Academic Press, Inc., Boston, MA, 1989).

[26] L. Puig, Nilpotent blocks and their source algebras, Invent. Math. 93 (1988), 77-116.

[27] L. Puig and A. Watanabe, On blocks with one simple module in any Brauer correspondenct, J. Algebra 163(1994), 135-138.

[28] M. Sawabe and K. Uno, Conjectures on character degrees for the simple Lyons group, Quart. J. Math. 54 (2003), 103-121.

[29] K. Uno, Conjectures on character degrees for the simple Thompson group, Osaka J. Math. 41 (2004), 11-36.

[30] K. Uno, Dade's conjecture for tame blocks, Osaka J. Math. 31 (1994), 747-772.

[31] A. Watanabe, On nilpotent blocks of finite groups, J. Algebra 163(1994), 128-134.

Jianbei An, Department of Mathematics, University of Auckland, Auckland, New Zealand

Charles W. Eaton, School of Mathematics, University of Manchester, United Kingdom Email: an@math.auckland.ac.nz_ｃharles.eaton@manchester.ac.uk 